\title{
Multiple myeloma-derived MMP-13 mediates osteoclast fusogenesis and osteolytic disease
}

\author{
Jing Fu, ${ }^{1}$ Shirong Li, ${ }^{1}$ Rentian Feng, ${ }^{2}$ Huihui Ma, ${ }^{3}$ Farideh Sabeh, ${ }^{4}$ G. David Roodman,,${ }^{5,6}$ Ji Wang, ${ }^{7}$ Samuel Robinson, ${ }^{7}$ \\ X. Edward Guo, ${ }^{7}$ Thomas Lund, ${ }^{8}$ Daniel Normolle, ${ }^{9}$ Markus Y. Mapara, ${ }^{1,3}$ Stephen J. Weiss, ${ }^{4}$ and Suzanne Lentzsch ${ }^{1,2}$ \\ 'Department of Medicine, Columbia University Medical Center, New York, New York, USA. ${ }^{2}$ University of Pittsburgh School of Medicine, Pittsburgh, Pennsylvania, USA. ${ }^{3}$ Columbia Center for Translational \\ Immunology, New York, New York, USA. 르epartment of Internal Medicine, Life Sciences Institute, University of Michigan, Ann Arbor, Michigan, USA. ${ }^{5}$ Department of Medicine, Indiana University, \\ Indianapolis, Indiana, USA. ${ }^{6}$ VA Medical Center, Indianapolis, Indiana, USA. ’Department of Biomedical Engineering, Columbia University, New York, New York, USA. ${ }^{8}$ Department of Hematology, \\ Odense University Hospital, Odense, Denmark. 'University of Pittsburgh Cancer Institute Biostatistics Facility, Pittsburgh, Pennsylvania, USA.
}

\begin{abstract}
Multiple myeloma ( $\mathrm{MM}$ ) cells secrete osteoclastogenic factors that promote osteolytic lesions; however, the identity of these factors is largely unknown. Here, we performed a screen of human myeloma cells to identify pro-osteoclastogenic agents that could potentially serve as therapeutic targets for ameliorating MM-associated bone disease. We found that myeloma cells express high levels of the matrix metalloproteinase MMP-13 and determined that MMP-13 directly enhances osteoclast multinucleation and bone-resorptive activity by triggering upregulation of the cell fusogen DC-STAMP. Moreover, this effect was independent of the proteolytic activity of the enzyme. Further, in mouse xenograft models, silencing MMP-13 expression in myeloma cells inhibited the development of osteolytic lesions. In patient cohorts, MMP-13 expression was localized to BM-associated myeloma cells, while elevated MMP-13 serum levels were able to correctly predict the presence of active bone disease. Together, these data demonstrate that MMP-13 is critical for the development of osteolytic lesions in MM and that targeting the MMP-13 protein - rather than its catalytic activity - constitutes a potential approach to mitigating bone disease in affected patients.
\end{abstract}

\section{Introduction}

Multiple myeloma ( $\mathrm{MM}$ ) arises as a consequence of the clonal expansion of malignant plasma cells within the BM compartment, an event associated with bone destruction and significant comorbidities including severe pain, pathologic fractures, and spinal cord compression (1). MM cells are commonly found in association with sites of active bone resorption, where they secrete a variety of pro-osteoclastogenic agents $(1,2)$. Current agents such as biphosphonates for the treatment of MM bone disease are aimed at specifically targeting osteoclast (OCL) function (3). However, these therapeutics only partially inhibit the progression of osteolytic lesions (3). Consequently, new treatment strategies that modulate MM-OCL interactions are needed to improve patient outcomes. While recent studies have identified several MM-derived factors that contribute to OCL activation, including receptor activator of NF- $\kappa$ B ligand (RANKL) (4), macrophage inflammatory protein $1 \alpha$ $(\mathrm{MIP}-1 \alpha)(5)$, and IL-3 (6), preliminary efforts aimed at targeting these molecules have not ameliorated MM-associated bone disease to a significant degree (7), suggesting that other key, but heretofore unknown, factors are operative at the MM-OCL interface.

While screening for osteoclastogenic factors secreted by MM cells, we unexpectedly found that the matrix metalloproteinase MMP-13 (alternatively termed collagenase-3) is highly expressed

Authorship note: J. Fu and S. Li are co-first authors and contributed equally to this work. Conflict of interest: The authors have declared that no conflict of interest exists. Submitted: December 29, 2014; Accepted: February 18, 2016.

Reference information: J Clin Invest. 2016;126(5):1759-1772. doi:10.1172/JCI80276. by human MM cells. MMP-13 is a member of a family of zinc- and calcium-dependent proteolytic enzymes that degrade extracellular matrix components during normal physiological processes (e.g., embryonic development, reproduction, tissue remodeling, and angiogenesis) as well as in pathological conditions such as osteoarthritis, tumor invasion, and metastasis $(8,9)$. In each of these scenarios, the key functional activities of MMP-13 have been linked directly to its ability to degrade interstitial collagen, a critical structural component of all connective tissues, including bone, as well as other matrix-associated targets (10-19). However, in contrast to studies highlighting the degradative activities of the metalloproteinase, we now report that MM-derived MMP-13 serves as a potent secretagogue of OCL fusion and bone-resorptive activity independently of its proteolytic activity by triggering the ERK1/2-dependent regulation of nuclear factor of activated $\mathrm{T}$ cells, cytoplasmic, calcineurin-dependent 1 (NFATc1) and dendrocyte-expressed 7 transmembrane (DC-STAMP) expression. These findings, coupled with data supporting enhanced MMP-13 expression in patients with MM, provide the first proof to our knowledge of a nonproteolytic role for MMP-13 in the development of lytic bone lesions in patients with $\mathrm{MM}$, thereby providing a rationale for targeting an unexpected effector that is operative within the MM-OCL interface.

\section{Results}

MMP-13 expression and regulation in $M M$ cells. While screening human MM cell lines for their ability to express MMPs, RPMI 8266 cells were noted to secrete MMP-13 at levels that far exceeded those 
A

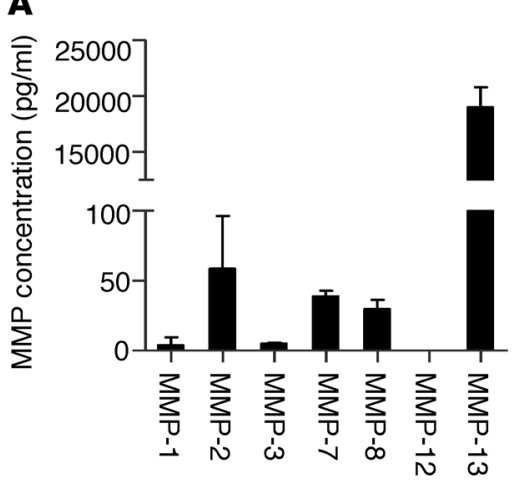

B

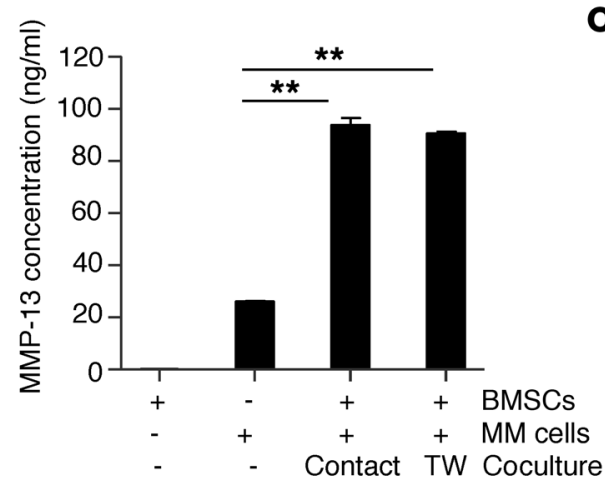

C

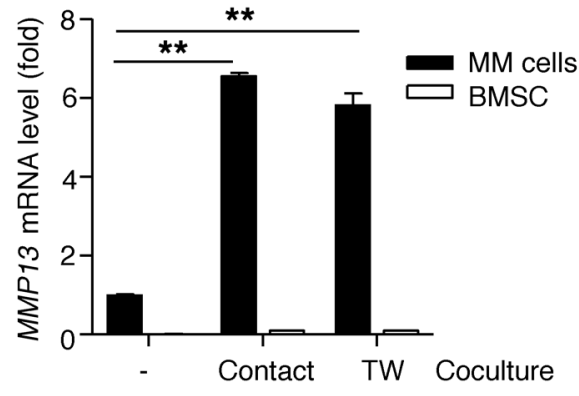

D
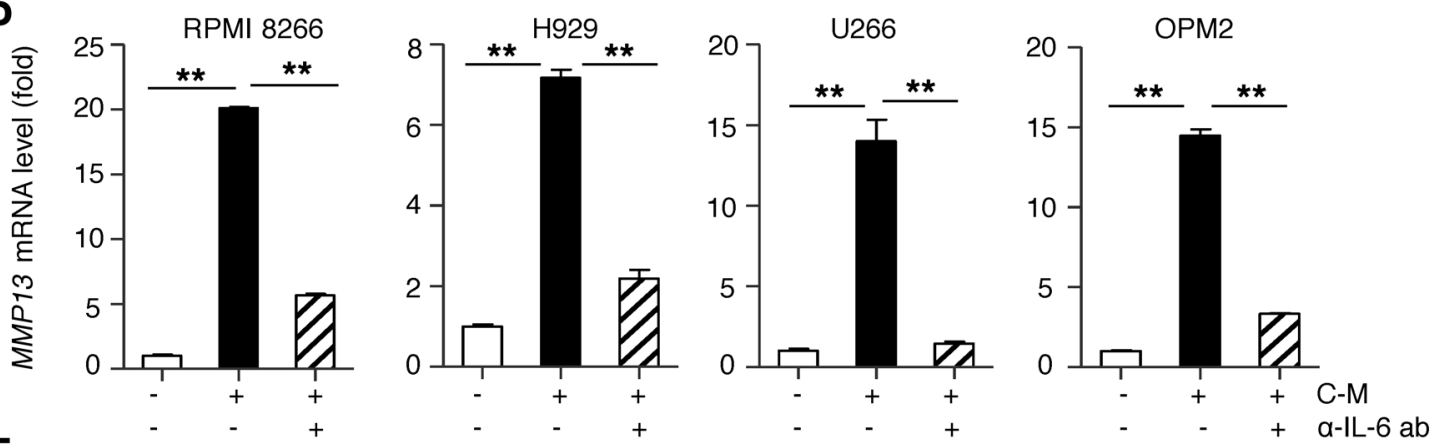

E
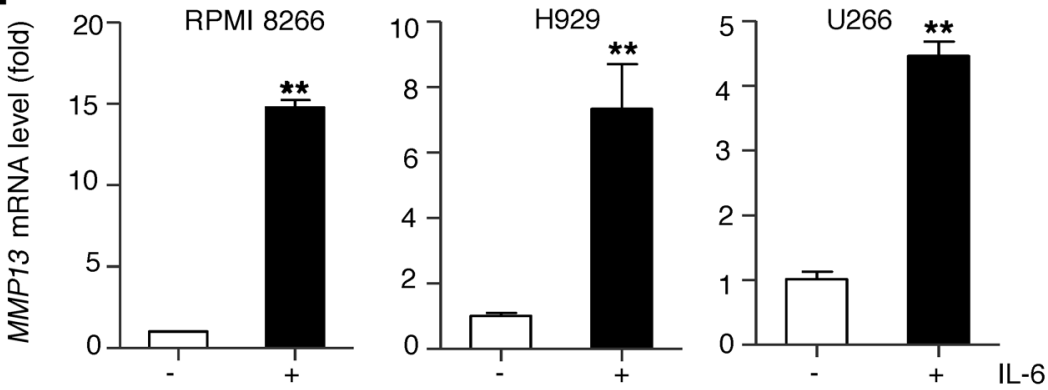

$\mathbf{F}$

RPMI 8266
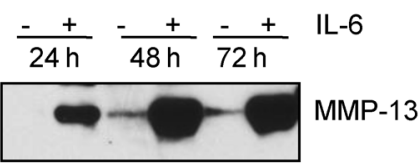

Culture SN

Figure 1. Cell-autonomous and BMSC-dependent regulation of MMP-13 in MM cells. (A) MMP protein expression levels in human RPMI 8266 MM cells as determined by Luminex assay (mean $\pm S D ; n=3$ ). (B) BMSCs were isolated from patients with MM and cultured with RPMI 8226 cells in direct contact (Contact) or in Transwell dishes (TW). MMP-13 concentrations in cell-free supernatants were determined by immunoassay (mean \pm SD; $n=3$ ). Data are representative of 2 independent experiments. ${ }^{* *} P \leq 0.01$, by ANOVA. (C) GFP+ RPMI 8226 cells were cocultured with BMSCs in direct contact or in Transwell dishes. MMP13 mRNA expression in RPMI 8226 cells was determined by qRT-PCR after separating GFP- BMSCs and GFP ${ }^{+}$RPMI 8266 cells by flow cytometry. Data represent the mean \pm SD $(n=3)$ and are representative of 2 independent experiments. ${ }^{* *} P \leq 0.01$, by ANOVA. (D) MM cell lines were cultured with or without CM prepared from BMSCs and a neutralizing Ab directed against IL-6 $(5 \mu \mathrm{g} / \mathrm{ml})$ for 24 hours. MMP13 mRNA levels were determined by qRT-PCR. Data represent the mean $\pm S D(n=3)$ and are representative of 3 independent experiments. ${ }^{* *} P \leq 0.01$, by ANOVA. (E) MM cells were treated with IL- 6 (20 $\mathrm{ng} / \mathrm{ml}$ ) for 24 hours, and MMP13 mRNA levels were determined by qRT-PCR. Data represent the mean \pm SD $(n=3)$ and are representative of 3 independent experiments. ${ }^{* *} P \leq 0.01$, by 2 -tailed Student's $t$ test. (F) RPMI 8266 cells were treated with IL- $6(20 \mathrm{ng} / \mathrm{ml})$ for the indicated durations, and MMP-13 protein levels were analyzed in culture supernatants (SN) by Western blotting. Data are representative of 2 independent experiments.

of other MMPs (e.g., MMP-1, -2, -3, -7, -8, and -12). Though MMP$1,-2,-3,-7$, and -8 levels all fell within detectable ranges, MMP-13 expression was approximately 300- to 400-fold higher than that of all other MMPs assessed (Figure 1A). Further, increased MMP13 expression was not confined to RPMI 8266 cells and could be detected similarly in all tested human MM cell lines, as determined by either MMP-13 fluorimetric assay (Supplemental Figure 1A; supplemental material available online with this article; doi:10.1172/ JCI80276DS1) or collagen zymography (Supplemental Figure 1B). Of note, when assessed by zymography, only trace amounts of the active form, as opposed to the pro-form, of MMP-13 were detected (Supplemental Figure 1B).
In vivo, BM stromal cells (BMSCs) are known to support MM cell growth and survival via both contact-dependent and -independent mechanisms $(20,21)$. Hence, we next determined whether BMSCs contribute to MMP-13 upregulation in MM cells. For this purpose, $\mathrm{GFP}^{+}$RPMI 8266 cells were cocultured with BMSCs in direct contact or, alternatively, in Transwell dishes, thereby precluding direct cell-cell contact. Under both coculture conditions, MMP-13 was significantly upregulated relative to that detected in supernatants from MM or BMSC cultures alone (Figure 1B), demonstrating that direct cell contact is not required for MMP-13 upregulation. Further, MM cells are the major source of MMP-13 production in the coculture system, as the MMP13 mRNA 

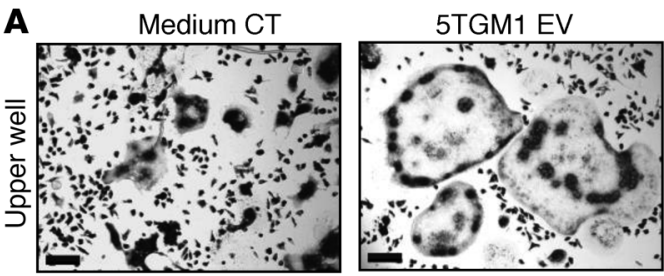

5TGM1 MMP-13-KD

5TGM1 MMP-13-KD
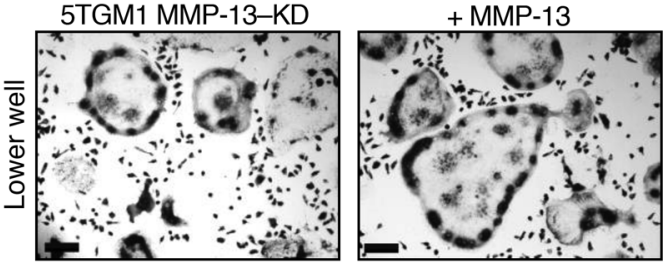

C
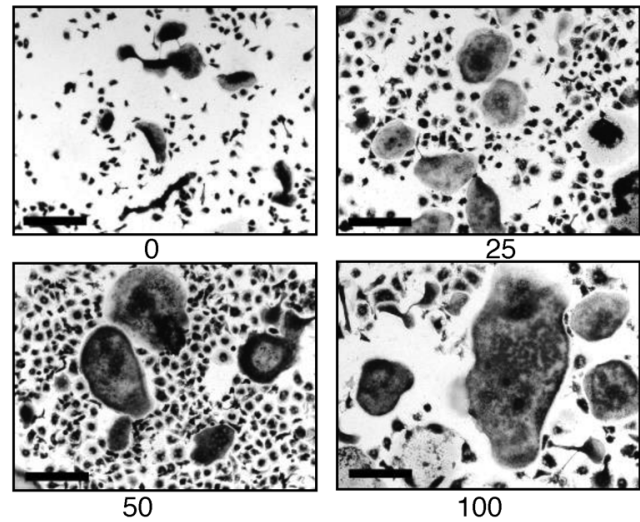

E

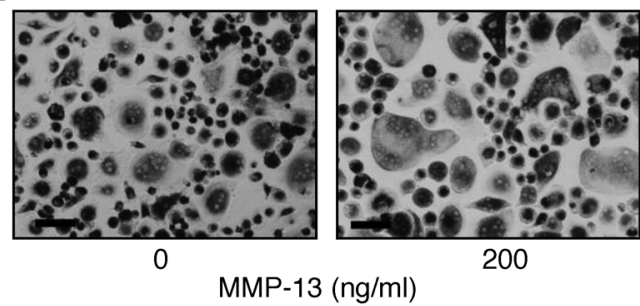

$\mathbf{B}$
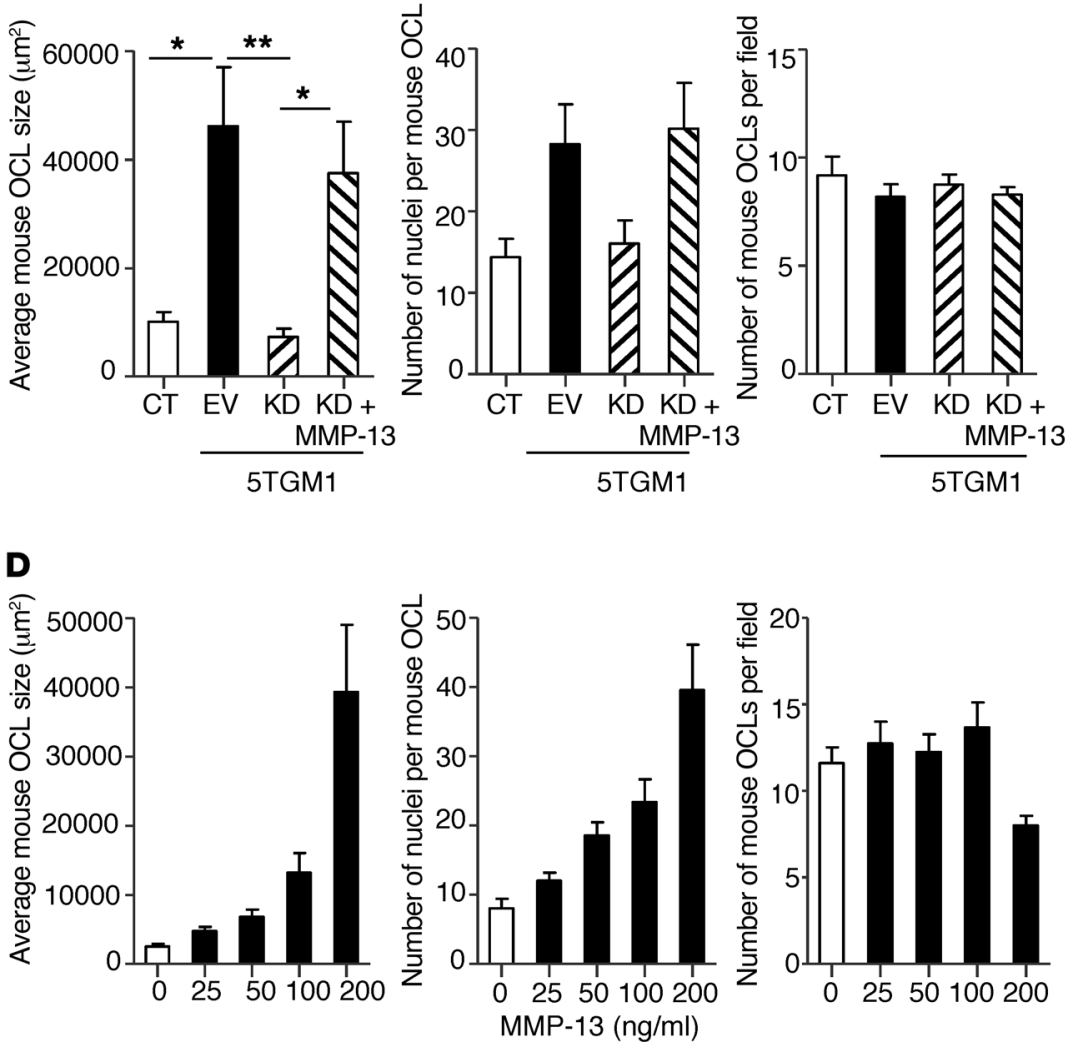

कै
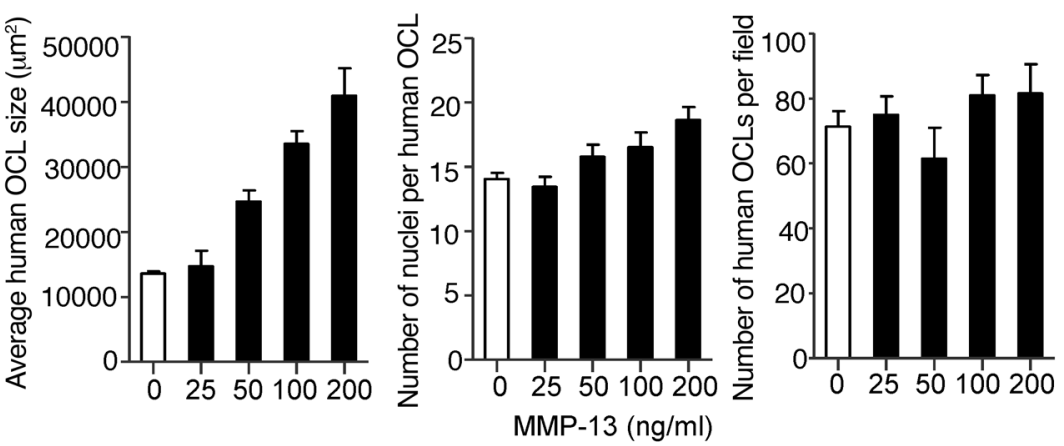

Figure 2. MMP-13 mediates MM-induced OCL fusion and bone resorption. (A) Mouse nonadherent BM cells were cocultured with 5TCM1-EV or 5TCM1MMP-13-KD \#1 cells in Transwell dishes with M-CSF (10 ng/ml) and RANKL (50 ng/ml) for 4 days, with pro-MMP-13 added as indicated. OCLs were stained for TRAP and images captured as described in Methods. Scale bars: $100 \mu \mathrm{m}$. Data are representative of 3 independent experiments. CT, control. (B) Mouse OCL size (left panel), number of nuclei per OCL (middle panel), and number of OCLs per field (right panel) were assessed by microscopy and Imagej software $(\mathrm{NIH})$. Data represent the mean $\pm \operatorname{SEM}(n=3)$. ${ }^{*} P \leq 0.05$ and ${ }^{*} P \leq 0.01$, by ANOVA. (C) Mouse nonadherent BM cells were cultured with the indicated concentrations of pro-MMP-13 or vehicle during OCL formation in the presence of M-CSF $(10 \mathrm{ng} / \mathrm{ml})$ and RANKL ( $50 \mathrm{ng} / \mathrm{ml}^{2}$ ). TRAP' OCLs were scored microscopically. Scale bars: $100 \mu \mathrm{m}$. Data are representative of 4 independent experiments. (D) Mouse OCL size (left panel), number of nuclei per OCL (middle panel), and number of OCLs per field (right panel) were determined as above. Data represent the mean \pm SEM $(n=3)$. $P \leq 0.0001$, by linear regression (left panel) and Poisson regression (middle panel); $P>0.05$ by Poisson regression (right panel). (E) Human mononuclear BM cells were cultured with the indicated concentrations of MMP-13 or vehicle during OCL formation in the presence of M-CSF $(10 \mathrm{ng} / \mathrm{ml})$ and RANKL ( $50 \mathrm{ng} / \mathrm{ml})$. OCL numbers were determined by CD51/CD61 staining with 23c6 Ab and scored microscopically. Scale bars: $100 \mu \mathrm{m}$. Data are representative of 3 independent experiments. (F) Human OCL size, number of nuclei per OCL, and number of OCLs per well (right panel) were determined as above. Data represent the mean $\pm \mathrm{SEM}(n=3)$. $P \leq 0.0001$, by linear regression (left panel) and Poisson regression (middle panel); $P>0.05$ by Poisson regression (right panel).

level in purified $\mathrm{GFP}^{+}$RPMI 8266 cells was more than 100-fold higher than that detected in BMSCs (Figure 1C). Similar results were obtained when any of the panel of human MM cell lines were incubated with BMSC-conditioned medium (CM). Further, the upregulation of MMP-13 could be almost completely blocked by adding a neutralizing Ab against IL-6 to the cultures (Figure 1D), suggesting that IL-6 is the key cytokine that induces MMP-13 in BMSC CM. In accordance with this finding, direct treatment of MM cells with exogenous IL-6 upregulated MMP13 transcription and protein expression (Figure 1, E and F). 
MMP-13 mediates MM-induced OCL fusion and bone resorption. To assess the potential impact of MM-derived MMP-13 on OCL function, mouse BM mononuclear cells (the source of OCL progenitors) were cultured in the lower compartment of Transwell dishes with murine 5TGM1 MM cells seeded in the upper compartment in the absence or presence of IL-6. Either IL-6 or 5TGM1 cells alone stimulated OCL formation (Supplemental Figure 2, A and B). However, in the presence of both IL-6 and 5TGM1 cells, OCL formation was significantly enhanced, both in terms of increased average size and nuclei numbers (Supplemental Figure 2, A and B), indicating that IL-6-stimulated MM cells secreted soluble factors that are capable of promoting OCL fusion and activity (2). To determine the degree to which MMP-13 participates in pro-osteoclastogenic activity, the metalloproteinase was silenced in mouse 5TGM1 MM cells by lentiviral shRNA transduction (confirmed by quantitative reverse transcription PCR [qRT-PCR]; Supplemental Figure 3). While coculture with 5TGM1 empty vector-transduced (EV-transduced) cells significantly increased OCL fusion relative to medium alone, 5TGM1-MMP-13-knockdown \#1 (5TGM1-MMP-13-KD \#1) cells largely lost the ability to induce OCL fusion, as reflected in the decreased OCL size and nuclei numbers. Underlining the specificity of the silencing strategy, OCL fusion was rescued by exogenous pro-MMP-13 (Figure 2, A and B).

A required role for MMP-13 in controlling 5TGM1-induced osteoclastogenesis raised the possibility that the proteinase either modulates MM function or acts as an osteoclastogenic factor. In OCL formation assays, pro-MMP-13 directly enhanced mouse (Figure 2, C and D) as well as human OCL formation in a concentration-dependent manner (Figure 2, E and F). As observed in the intact MM cell model, pro-MMP-13 increased both OCL size and nuclei numbers (Figure 2, D and F) without affecting pre-OCL proliferation (Supplemental Figure 4A). As a result of increased fusion, we observed a trend toward decreased OCL numbers (Figure 2D and Supplemental Figure 2B). Time course assays adding MMP-13 on days 1 and 2 only, on days 3 and 4 only, or on days 1-4 of OCL culture revealed that the presence of MMP- 13 is critical during the entire culture period in order to induce OCL fusion (Supplemental Figure 4B), without significant effects on cell proliferation (Supplemental Figure 4C). Consistent with the increase in OCL size and nuclei numbers, the bone-resorptive activity of OCLs also increased as a function of MMP-13 concentration (Supplemental Figure 4, D and E). By contrast, supplementing cultures with either MMP-1, MMP-2, or MMP-9, three other MMPs whose expression has been linked previously to tumor-related bone metastasis (22), exerted no significant effects on OCL development (Supplemental Figure 5, A and B).

The ability of exogenous MMP-13 to support osteoclastogenesis as well as OCL activity raised the possibility that OCL progenitors may express MMP-13 as an endogenously derived pro-osteoclastogenic agent. Indeed, WT, but not $\mathrm{Mmp13}^{-1-}$, BM-derived mononuclear cells expressed Mmp13 as assessed by qRT-PCR (Supplemental Figure 6A). Furthermore, OCL formation from $\mathrm{Mmp13}^{-/-} \mathrm{BM}$-derived mononuclear cells was significantly impaired relative to that observed in WT cells, as evidenced by significant decreases in OCL size, nuclei numbers (Supplemental Figure 6, B and C), and bone-resorptive activity (Supplemental Figure 6, D and E). As predicted, defects in Mmp13-/- OCL forma- tion were reversed by supplementing the cultures with exogenous pro-MMP-13 (Supplemental Figure 6, B and C), without affecting pre-OCL proliferation rates (Supplemental Figure 6F). Taken together, these data indicate that MMP-13 modulates OCL fusion and bone-resorptive activity in both a cell-autonomous and a noncell-autonomous manner.

MMP-13 induces OCL formation independently of its proteolytic activity by an ERK1/2-DC-STAMP-dependent process. MMP-induced activities are most frequently associated with the conversion of the proenzyme to a mature form that is able to hydrolyze target substrates $(8,9)$. Hence, as the MMP-13 zymogen enhanced OCL fusion and function in our in vitro cultures, we sought to determine (a) whether pro-MMP-13 is processed into its catalytically active form by BM-derived mononuclear cells and (b) whether proteolytic activity is required for the induction of OCL formation. Unexpectedly, the conversion of the MMP- 13 zymogen to its active form was not detected in $\mathrm{BM}$ mononuclear cell cultures in the $\mathrm{CM}$ (Figure $3 \mathrm{~A}$, upper panel) or in association with the OCL surface (Figure $3 \mathrm{~A}$, lower panel), suggesting that MMP-13 enzymatic activity is not necessarily required for the stimulation of osteoclastogenic activity. Indeed, neither of the 2 specific and potent MMP-13 enzymatic inhibitors CP544439 $\left(\mathrm{IC}_{50}=0.7 \mathrm{nM}\right.$ ) (23) or CAS 544678-85-5 $\left(\mathrm{IC}_{50}=8 \mathrm{nM}\right)(24)$ affected MMP-13-induced OCL formation (Figure 3, B and C) at concentrations that completely blocked MMP-13 enzymatic activity (Supplemental Figure 7A).

To confirm that the proteolytic activity of MMP-13 is not required for induction of OCL fusion and activity, an enzymatically inactive mutant of MMP-13 was generated by introducing an E223A mutation within the zinc-binding motif of the proteinase (Figure $4 \mathrm{~A}$ and ref. 25). As expected, the MMP-13 WT protein rapidly underwent autoactivation in the presence of $p$-aminophenylmercuric acetate (a process dependent on an intact catalytic domain), while the MMP-13 E223A mutant remained locked in its proform state, as confirmed by a fluorometric assay that showed an absence of MMP-13 enzymatic activity (Figure 4B) or by collagen zymography (Supplemental Figure 7B). Nevertheless, both MMP$13 \mathrm{WT}$ and MMP-13 E223A showed similar abilities to promote OCL formation (Figure 4, C and D) and bone-resorptive activity (Figure 4, E and $\mathrm{F}$ ), confirming that MMP-13 proteolytic activity is not required for the induction of osteoclastogenesis. Following knockin of MMP-13 WT or MMP-13 E223A into 5TGM1 MMP-13KD cells (Supplemental Figure 8A), OCL induction was rescued in Transwell coculture assays (Supplemental Figure 8, B and C). While recent studies have suggested that proteinase-independent activities of selected MMPs may reside within the C-terminal hemopexin domain (26-30), neither the recombinant MMP-13 hemopexin nor the recombinant procatalytic domain alone (Supplemental Figure 9A) were able to reconstitute the activity of the full-length enzyme (Supplemental Figure 9, B and C).

DC-STAMP, a master fusogen essential for normal OCL function, is induced by RANKL via $\operatorname{NFATc1}(31,32)$. Consistent with the MMP-13-dependent inductive activity of OCL fusion, both the WT and E223A mutant forms of the proteinase upregulated NFATc1 and DC-STAMP expression at both the protein and mRNA levels in developing OCLs (Figure 4G and Supplemental Figure 10, A and B). Flow cytometric staining confirmed the induction of cell-surface DC-STAMP expression by MMP-13 
A

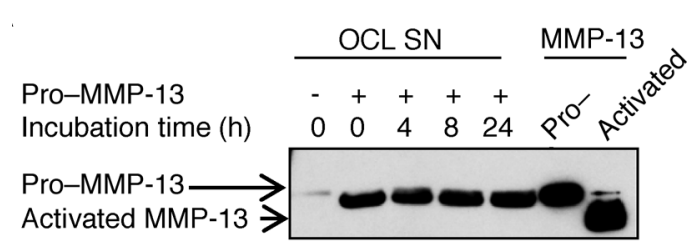

B

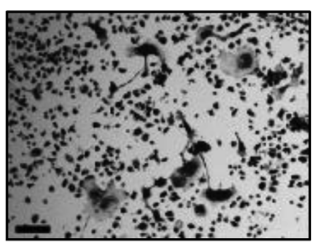

CT
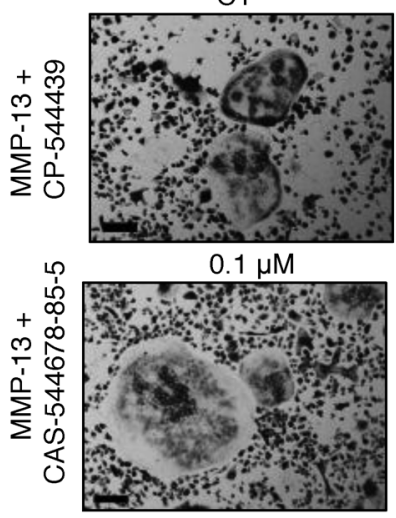

$0.1 \mu \mathrm{M}$

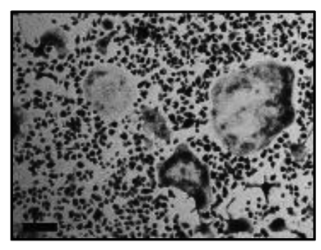

MMP-13

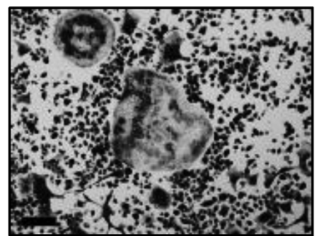

$1 \mu \mathrm{M}$

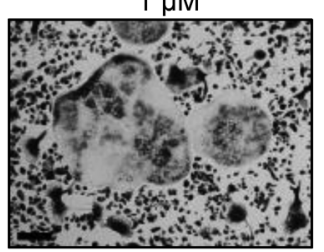

$1 \mu \mathrm{M}$

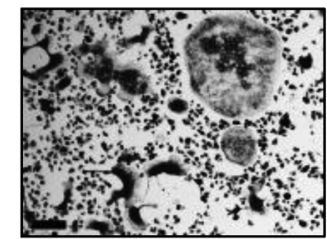

$10 \mu \mathrm{M}$

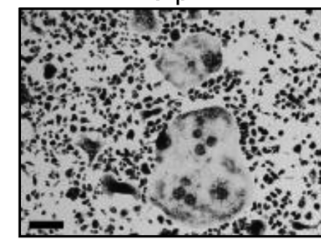

$10 \mu \mathrm{M}$
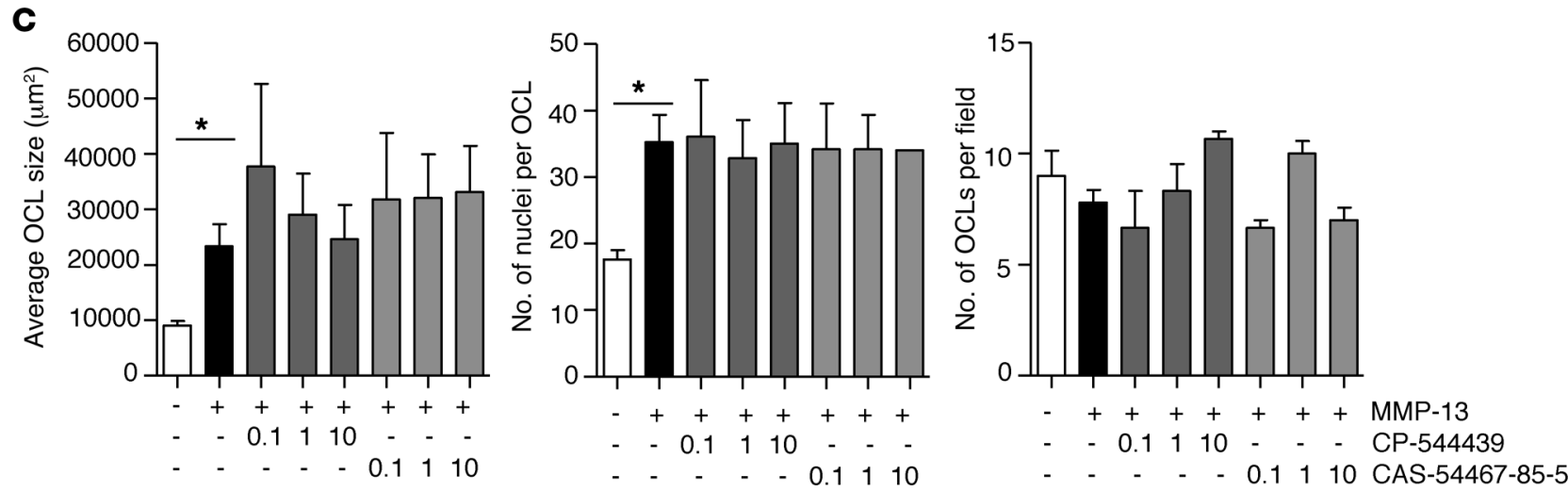

Figure 3. MMP-13-dependent osteoclastogenesis proceeds independently of pro-MMP-13 activation. (A) Mouse nonadherent BM cells were cultured for 4 days with M-CSF $(10 \mathrm{ng} / \mathrm{ml}$ ) and RANKL $(50 \mathrm{ng} / \mathrm{ml})$ to induce OCL differentiation in the absence or presence of pro-MMP-13 (200 ng/ml). Top panel: culture media were collected at the indicated times after the addition of pro-MMP-13. Processing of pro-MMP-13 to mature/active MMP-13 was assessed by Western blotting. Pro- and active recombinant MMP-13 protein standards were loaded as controls. Lower panel: OCL lysates isolated from cells incubated with or without pro-MMP-13 were analyzed by Western blotting. Data are representative of 4 independent experiments. WCL, whole-cell lysate. (B) Mouse nonadherent BM cells were cultured as described in $\mathbf{A}$, in the absence or presence of pro-MMP-13 and the MMP-13 synthetic inhibitor CP544439 or CAS 544678-85-5, at the indicated concentrations. OCL formation was assessed by TRAP staining. Scale bars: $100 \mu$ m. Data are representative of 3 independent experiments. (C) OCL size, number of nuclei per OCL, and number of OCLs per field in B were determined by microscopy and Imagej analysis. Data represent the mean $\pm \operatorname{SEM}(n=3) .{ }^{*} P \leq 0.05$, by ANOVA.

(Supplemental Figure 10C and refs. 33, 34). Consistent with these findings, $M m p 13^{--}$OCLs also generated lower expression levels of NFATc1 and DC-STAMP, both of which were partially rescued by exogenous MMP-13 (Supplemental Figure 11). Interestingly, MMP-13 has recently been reported to activate ERK1/2 signaling in chondrocytes (35). As ERK1/2 can serve as a potential regulator of the NFATc1/DC-STAMP axis (36), we sought to determine whether MMP-13 drives osteoclastogenesis through this signal transduction cascade. Indeed, both MMP-13 WT and the E223A mutant directly activated ERK signaling in BM-derived pre-OCLs (Supplemental Figure 12A). Further mechanistic studies indicated that both MMP-13 WT and the E223A mutant upregulated TRAF6 polyubiquitination (ref. 37 and Supplemental Figure 12B) and induced phosphorylation of the ERK upstream kinases c-Raf and MEK1/2, as well as the downstream target substrate p90RSK (refs. 38, 39, and Supplemental Figure 12C), indicating that MMP13 upregulates ERK signaling via an enzymatic activity-independent mechanism. More important, following the inhibition of ERK signaling with U0126 (40), the ability of either MMP-13 WT or the E223A mutant to induce NFATc1 and DC-STAMP upregulation and OCL fusion was blocked (Figure 4, G and $\mathrm{H}$ ). Taken together, these data indicate that pro-MMP-13 functions as a secreted activator of an ERK signal transduction cascade that induces both NFATc1 and DC-STAMP expression.

MMP-13 drives MM-induced osteolysis in vivo. To assess the role of MMP-13 in the development of MM-associated bone disease in vivo, we next used an intratibial 5TGM1 murine model that recapitulates myeloma tumor growth coupled with severe osteolysis (41). To this end, GFP-expressing 5TGM1 cells were lentivirally transduced with EV control or 2 distinct shRNA expression vectors 


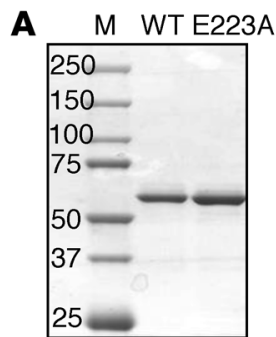

B
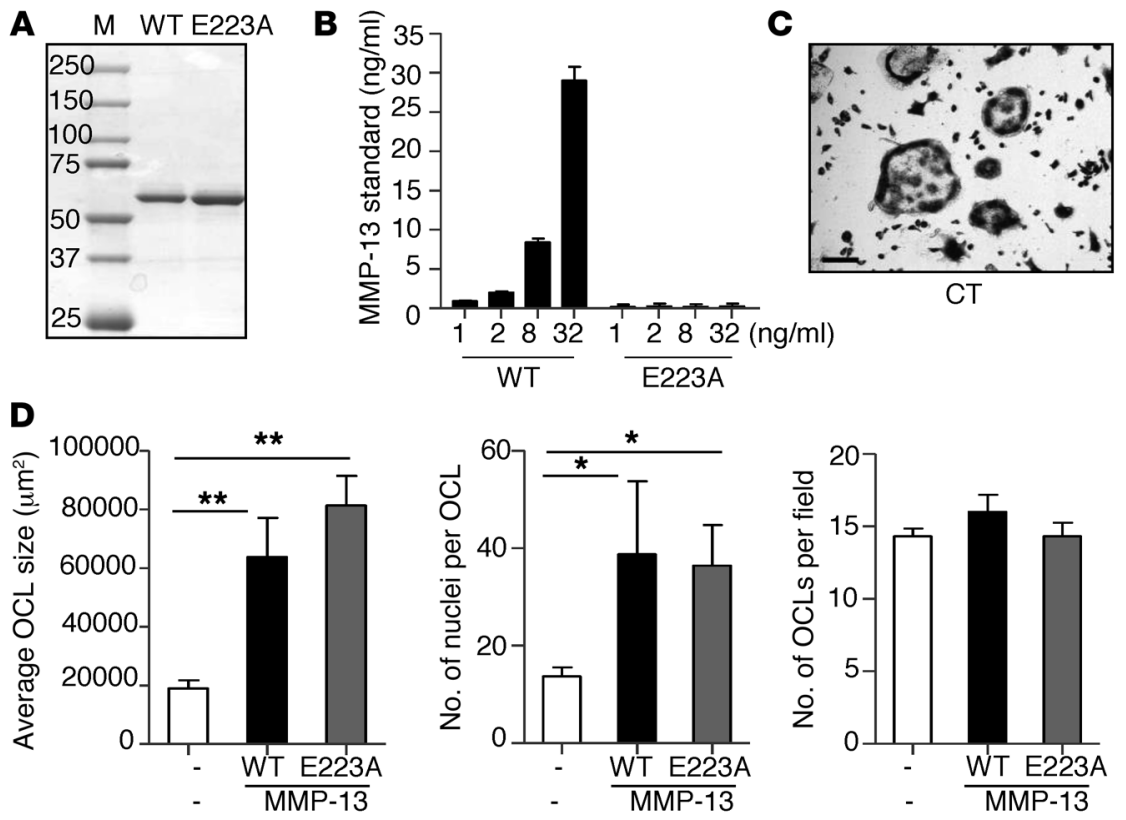

$\mathbf{F}$
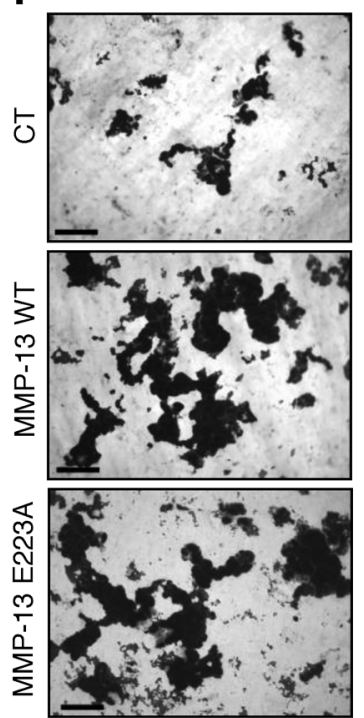
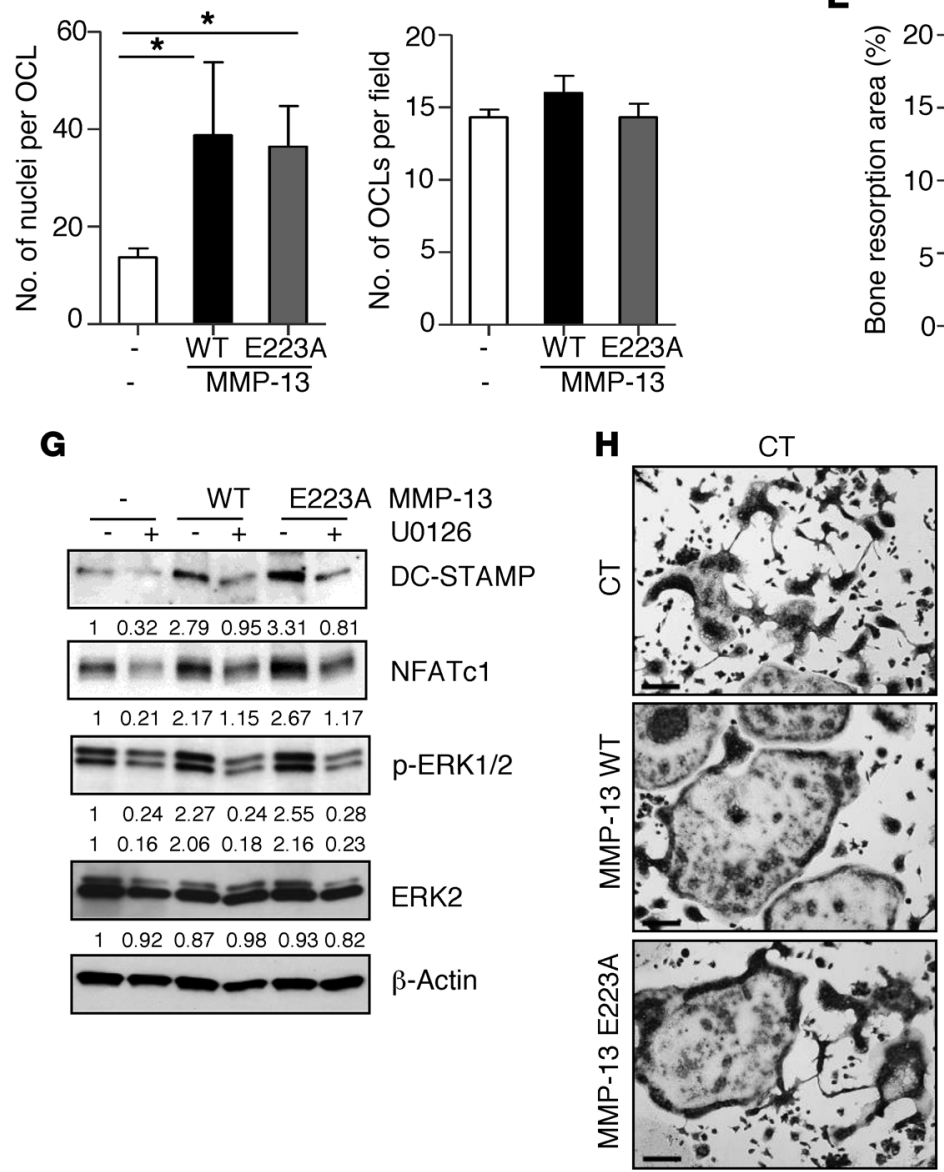

G

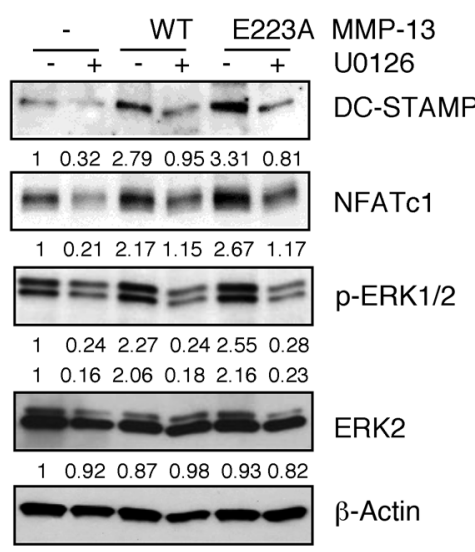

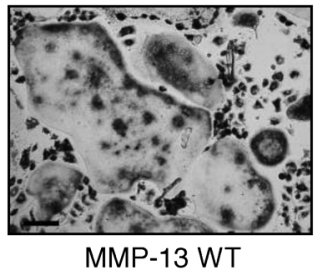

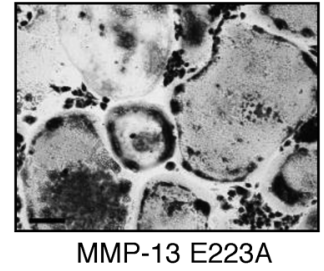

$\mathbf{E}$

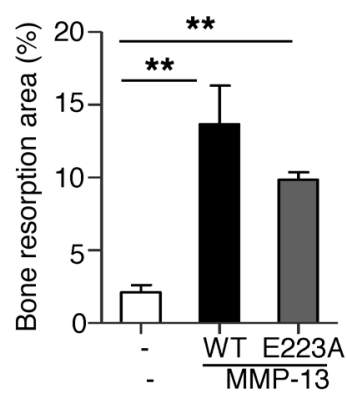

Figure 4. MMP-13 proteolytic activity is not required for OCL induction. (A) SDS-PAGE analysis of purified WT pro-MMP-13 and E223A pro-MMP-13. M, molecular weight marker. (B) Proteolytic activity of purified MMP-13 WT and E223A proteins was analyzed by fluorometric assay and compared with standard MMP-13 (mean \pm SD; $n=3$ ). Data are representative of 3 independent experiments. (C) For OCL induction, mouse nonadherent BM cells were cultured with WT pro-MMP-13 or the pro-MMP-13 E223A mutant in the presence of M-CSF (10 ng/ml) and RANKL (50 ng/ml) for 4 days. OCLs were stained for TRAP. Scale bar: $100 \mu \mathrm{m}$. Data are representative of 5 independent experiments. (D) OCL size, number of nuclei per OCL, and number of OCLs per field in C were determined as described above. Data represent the mean $\pm \operatorname{SEM}(n=3)$. ${ }^{*} P \leq 0.01$, by ANOVA (left panel) and ${ }^{*} P \leq 0.05$, by Poisson ANOVA (middle panel). (E and F) Mouse nonadherent BM cells were seeded atop dentin slices and cultured as described in $\mathbf{C}$ for 9 days. Bone resorption lacunae were visualized by hematoxylin staining. Bone resorption area was calculated by Image) software. Data represent the mean \pm SEM $(n=3)$ and are representative of 3 independent experiments. ${ }^{* *} P \leq 0.01$, by ANOVA $(\mathbf{E})$. Scale bars: $100 \mu \mathrm{m}(\mathbf{F})$. (G and $\left.\mathbf{H}\right)$ Mouse nonadherent BM cells were cultured as described in C, with or without pro-MMP-13 WT or the pro-MMP-13 E223A mutant, in the presence of U0126 (5 $\mu$ M) or vehicle control for 4 days. (G) DC-STAMP, NFATc1, p-ERK1/2, and ERK2 expression levels were determined in OCL lysates by Western blot analysis. Band intensities were quantified by Image software, normalized to $\beta$-actin, and compared with control. Relative intensities are shown. (H) OCL differentiation analyzed by TRAP staining. Data are representative of 2 independent experiments. Scale bars: $100 \mu \mathrm{m}$.

directed against MMP-13 (MMP-13-KD \#1 and MMP-13-KD \#2), thereby acting to efficiently silence metalloproteinase expression (confirmed by qRT-PCR and collagen zymography; Supplemental Figure 13, A and B), without affecting 5TGM1 cell proliferation (Supplemental Figure 13C). 5TGM1-GFP-EV, 5TGM1-GFP-MMP-
13-KD \#1, or 5TGM1-GFP-MMP-13-KD \#2 cells were injected intratibially into Rag2 ${ }^{--}$mice (41), and after a 4-week incubation period, tibiae were harvested for histological analysis, followed by micro-quantitative CT (micro-qCT). As shown in Figure 5, intratibial injection of 5TGM1-GFP-EV cells disrupted normal 

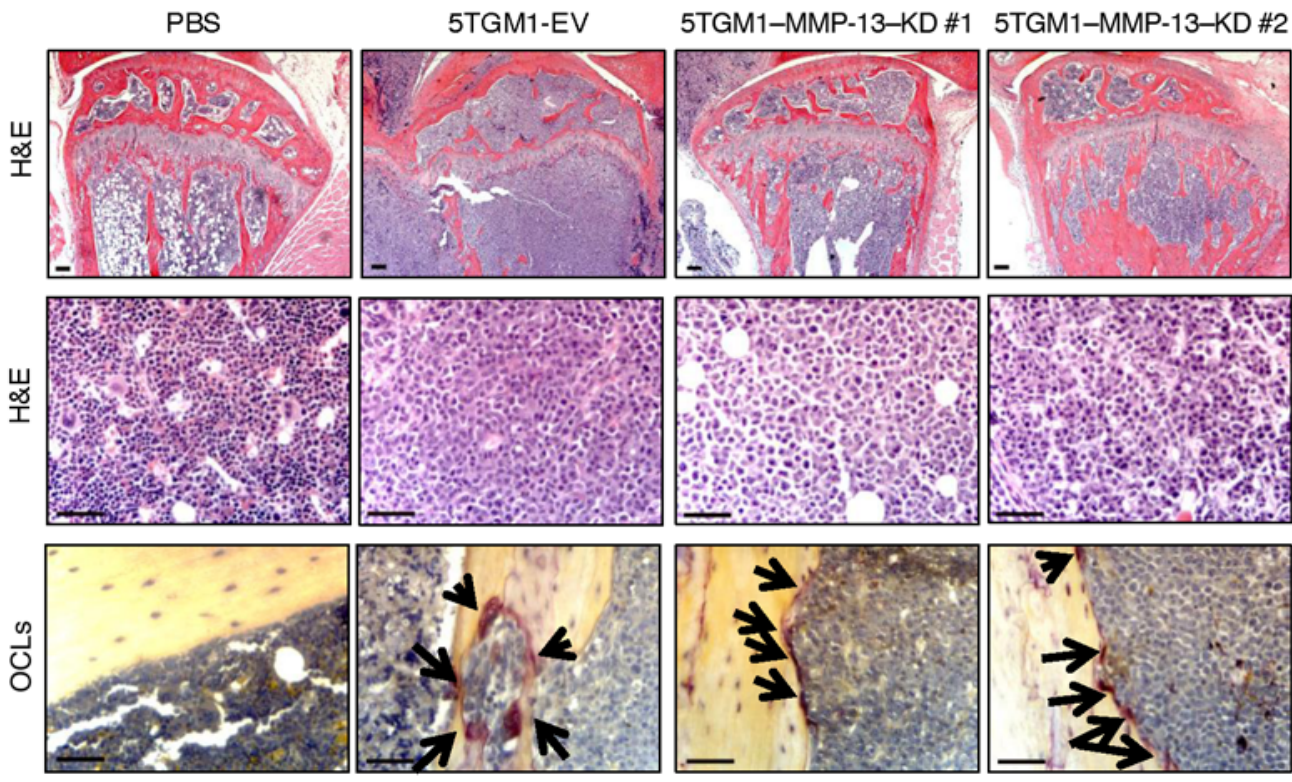

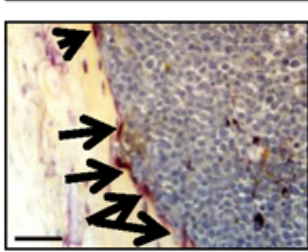

Figure 5. MMP-13 directs

$M M$-induced $O C L$ in vivo. $P B S$, 5TCM1-GFP-EV MM cells, 5TCM1GFP-MMP-13-KD \#1 MM cells, or 5TGM1-GFP-MMP-13-KD \#2 MM cells were injected bilaterally into both tibiae $(n=24)$ of $\operatorname{Rag}^{-1-}$ mice. Mice were sacrificed 4 weeks later and the tibiae formalin-fixed, followed by paraffin sectioning. Sections were then subjected to H\&E and TRAP staining. Representative images are shown for each set of conditions. Scale bars: $100 \mu \mathrm{m}$. hematopoiesis in association with the development of extensive lytic lesions, resulting in the destruction of trabecular bone. MMP13-KD \#1 and -KD \#2 MM cells also infiltrated the BM, but without completely replacing hematopoietic centers. Most important, in contrast to control 5TGM1-GFP-EV cells, bone structure was maintained with markedly less bone loss when recipient mice were inoculated with MMP-13-KD \#1 or -KD \#2 MM cells (Figure 5, top panel). As expected, while enlarged $\mathrm{TRAP}^{+}$OCLs were observed adjacent to the 5TGM1-GFP-EV tumor, only small TRAP ${ }^{+}$OCLs were detected in mice injected with 5TGM1-GFP-MMP-13-KD \#1 or $-\mathrm{KD} \# 2$ cells (Figure 5, bottom panel). Quantification of OCL numbers and size confirmed that MMP-13-KD decreased OCL size as determined by the OCL surface to bone surface ratio (Supplemental Figure 14).

Bone micro-qCT and morphological analysis of the tibiae and adjacent femurs demonstrated that 5TGM1-GFP-EV myeloma cells not only induced lytic bone lesions, but also resulted in trabecular bone loss (Figure 6A). By contrast, MMP-13-KD \#1 or -KD \#2 MM cells significantly inhibited the formation of MM-induced bone lesions (Figure 6A). These effects were further supported by our morphological analyses of trabecular bones (Figure 6B), in which MMP-13-KD \#1 or -KD \#2 MM cells significantly increased the bone volume fraction (bone volume/total volume [BV/TV]: EV 0.0573 vs. KD \#1, 0.0723 and $\mathrm{KD} \# 2,0.0987)$, connective density (Con.D.: EV 50.53 vs. KD \#1, 83.69 and KD \#2, $142.0 \mathrm{~mm}^{3}$ ), trabecular numbers (Tb.N.: EV 3.081 vs. KD \#1, 3.440 and KD \#2, 3.368 $\mathrm{mm}^{-1}$ ), and bone mineral density (BMD: EV 125.3 vs. KD \#1, 150.9 and $\mathrm{KD} \# 2,181.9 \mathrm{mg} / \mathrm{cm}^{3}$ ), while decreasing the bone surface to bone volume ratio (BS/BV: EV 74.44 vs. KD \#1, 71.53 and $\mathrm{KD} \# 2$, $64.01 \mathrm{~mm}^{-1}$ ) and the structure model index (SMI: EV 3.257 vs. KD $\# 1,3.023$ and $\mathrm{KD} \# 2,2.610$ ). Hence, silencing of MM-derived MMP-13 inhibits osteolysis in vivo, further confirming its critical role in MM bone disease.

In a second set of animal experiments, we focused on the effects of MMP-13 silencing on MM tumor progression. Four weeks after intratibial injection of 5TGM1-GFP-EV or 5TGM1-
GFP MMP-13-KD \#1 cells, serum levels of mouse IgG2b were measured to assess tumor burden, while tibiae were harvested for histological analysis as well as GFP imaging, followed by microqCT. Similarly, the BM infiltration of MMP-13-KD \#1 MM cells caused markedly less bone loss compared with that seen with 5TGM1-GFP-EV cells (Supplemental Figure 15A, top panel) and the formation of smaller OCLs in juxtaposition to MM cells (Supplemental Figure 15A, bottom panel). Despite the fact that MMP13 had no direct effect on MM cell growth, as demonstrated by in vitro ${ }^{3} \mathrm{H}$-thymidine incorporation assay (Supplemental Figure 15B), the decreased OCL activity and subsequent bone disease led to a slightly lower MM tumor burden in vivo, as reflected by quantitative fluorescence imaging (Supplemental Figure 15, C and D) and IgG2b secretion (Supplemental Figure 15E). Consistent with the previous results, micro-qCT analysis again showed that MMP13 silencing blocked the formation of MM-induced bone lesions in both the tibiae and adjacent femurs (Supplemental Figure 16).

MMP-13 expression in patients' plasma cells and serum. Given the key role played by MMP-13 in regulating MM-induced osteoclastogenesis in our model systems, we next sought to determine whether MMP-13 expression levels might serve as an index of bone disease in patients. MMP-13 expression was first monitored in marrow biopsies of a cohort of patients with MM and controls. In tissue arrays of 11 normal biopsy samples and $10 \mathrm{MM}$ samples, MMP-13 expression was specifically and highly expressed in primary MM BM samples (Figure 7A, bottom panel; 9 of 10 samples), but not in any of the normal control samples (Figure 7A, top panel; 0 of 11 samples). Given that MMP-13 is a secreted protein, we considered the possibility that serum levels of the metalloproteinase could reflect the presence of active bone disease. As such, we determined MMP-13 serum levels in 32 patients with MM and in 6 healthy donors. As shown in Figure 7B, 10 of 18 patients with $\mathrm{MM}$-associated bone disease showed positive serum expression levels of MMP-13, while none of the $14 \mathrm{MM}$ patients without bone disease had detectable levels of MMP-13. Further, MMP-13 was not detected in any of the 6 healthy donors. Thus, consistent 
A
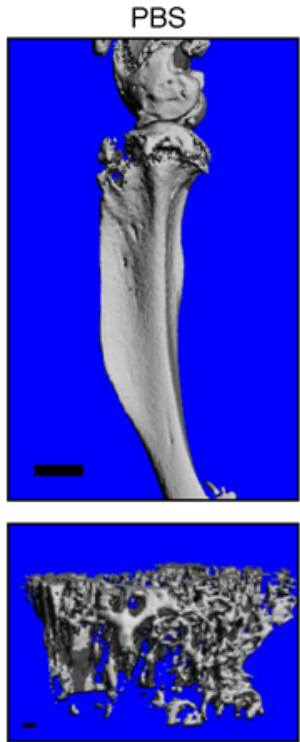

5TGM1-EV
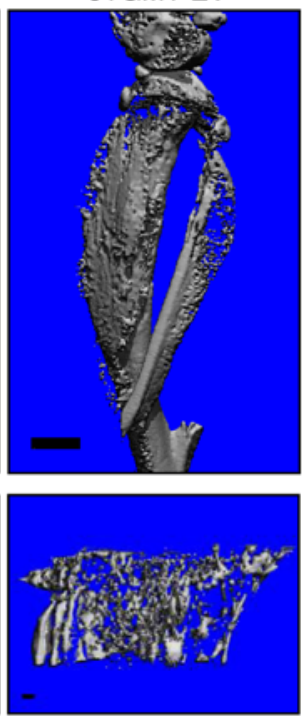

5TGM1-MMP-13-KD \#1 5TGM1-MMP-13-KD \#2
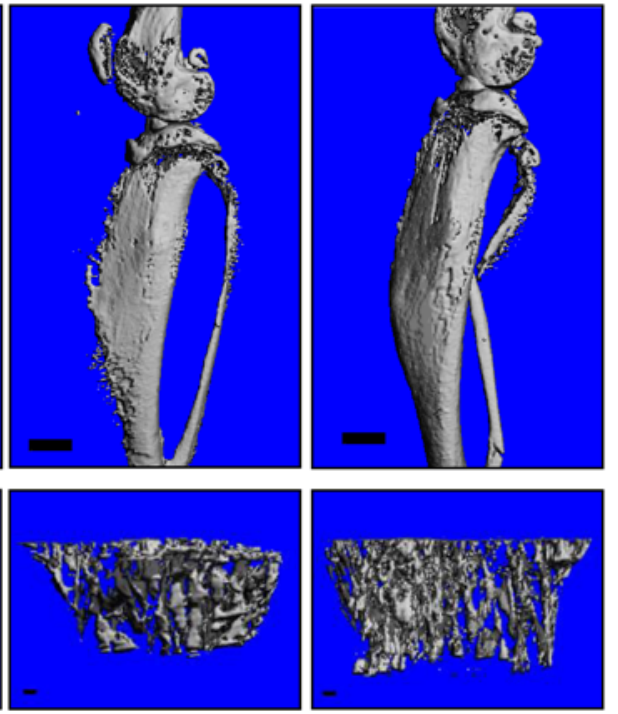
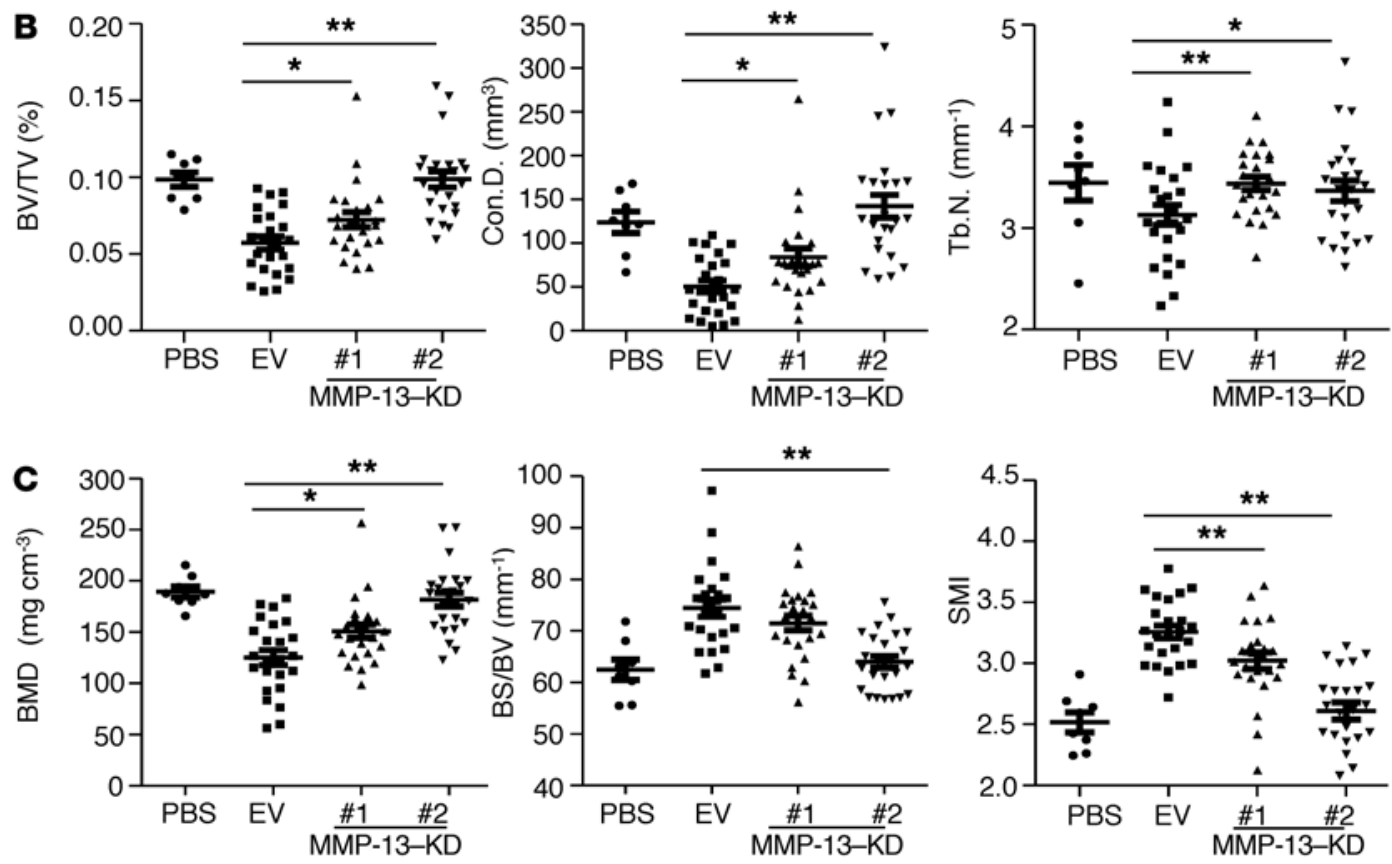

Figure 6. MMP-13 directs MM-induced osteolysis. (A) Tibiae from Figure 5 were analyzed by 3D micro-qCT scanning. Representative 3D images of tibiae, adjacent femurs (top panel), and tibiae trabecular bones (bottom panel) from each treatment regimen are shown. Scale bars: $1 \mathrm{~mm}$ (top panel); $100 \mu \mathrm{m}$ (bottom panel). (B and C) Trabecular bone of mice tibiae were analyzed, and microstructural parameters, including bone volume fraction (BV/TV), Con.D., Tb.N., BMD, BS/BV ratio, and SMI, were determined. Results are presented as the mean \pm SEM $(n=24) .{ }^{*} P \leq 0.05$ and ${ }^{* *} P \leq 0.01$, by ANOVA.

with a pivotal role for MMP-13 in MM-associated bone disease, serum MMP-13 levels were specific for bone disease status in a significant subset of affected patients.

\section{Discussion}

Following their homing to the BM compartment, MM cells secrete OCL activation factors that contribute to the formation of bony lesions in affected patients $(1-6,42)$. Here, we demonstrate that IL-6-mediated MM-BMSC interactions induce MMP-13 expression that, in turn, serves as a potent osteoclastogenic factor capable of promoting the generation of bone-resorptive, multinucle- ated OCLs. Unexpectedly, in contrast to previous studies that have sought to identify potential roles for MMPs in bone-resorptive disease states, MMP-13 exerts its pro-osteoclastogenic effects independently of a requirement for proteolytic activity by acting as a DC-STAMP-inducing secretagogue. Complementing these in vitro studies, the pathophysiological relevance of our findings are highlighted by the demonstration that silencing MMP-13 expression in MM cells exerts bone-sparing effects in a mouse MM disease model. In extending these findings to human populations, MMP-13 was readily detected at both the MM-BM interface and in the serum of a subset of patients with active bone disease. 
A
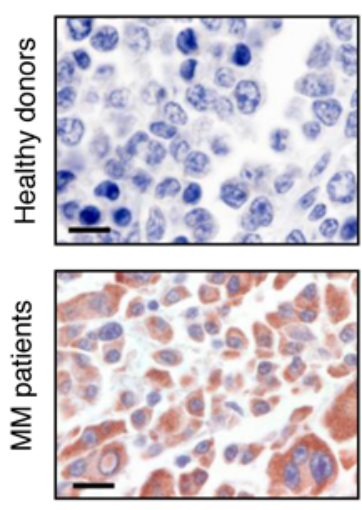
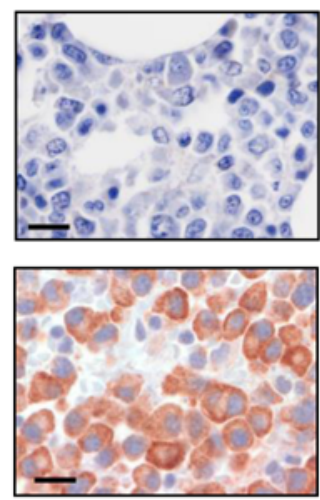

B

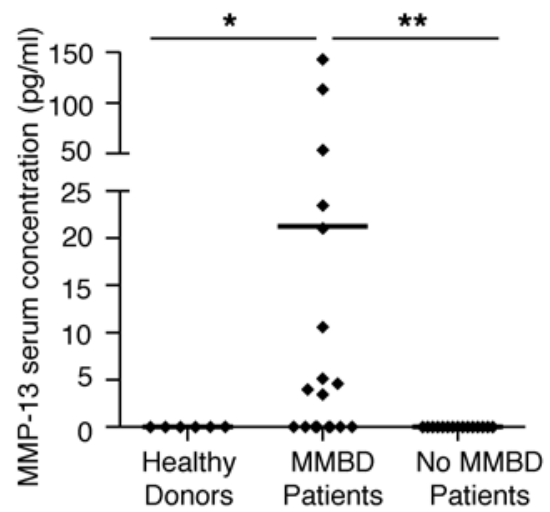

C

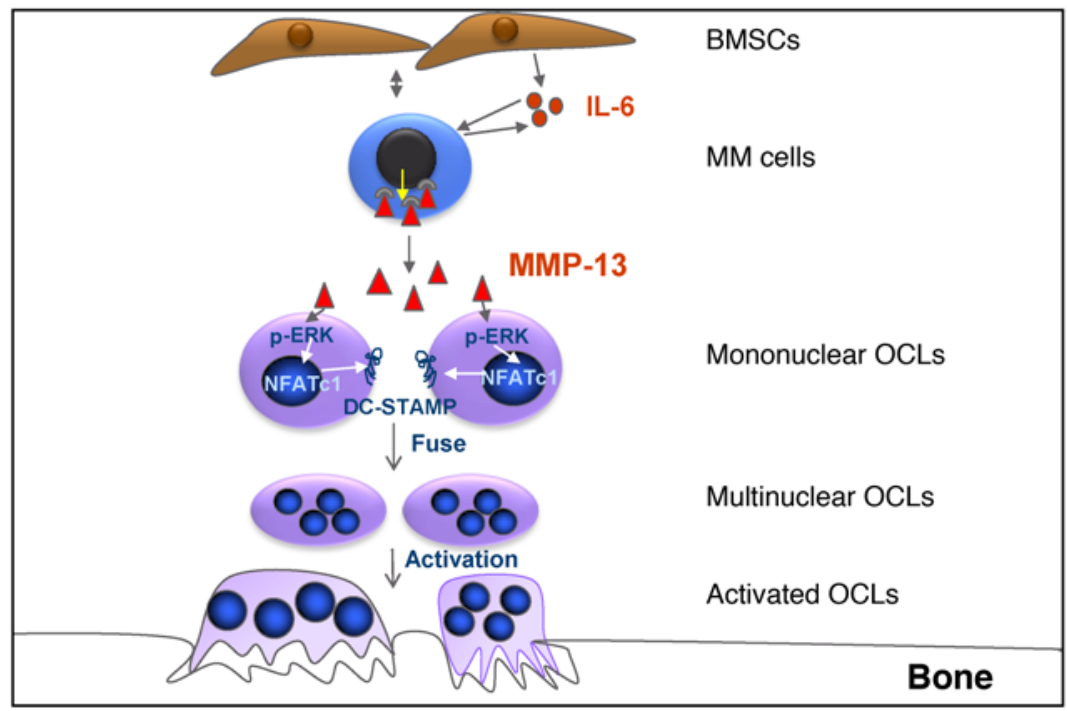

Figure 7. BM and serum MMP-13 levels in MM patients. (A) Tissue arrays of normal costal tissue (top panel, $n=10$ ) and of tissue from MM patients (bottom panel, $n=11$ ) were subjected to immunohistochemical staining using an hMMP13 Ab. Scale bars: $20 \mu \mathrm{m}$. (B) MMP-13 levels in sera from healthy donors $(n=6)$ and from MM patients with bone disease (MMBD) $(n=18)$ and without $\operatorname{MMBD}(n=14)$ were determined by ELISA. ${ }^{*} P \leq 0.05$ and ${ }^{*} P \leq 0.01$, by 2 Wilcoxon rank-sum tests with Bonferroni's correction. (C) Role of MMP-13 in MM: IL-6 expressed in the BM microenvironment induces MMP-13 secretion from MM cells. MMP-13 subsequently activates ERK1/2 signaling in OCL precursors and mediates the subsequent induction of NFATC1 and DC-STAMP expression, resulting in enhanced OCL fusion, activity, and osteolysis.
MM cells have long been known to express multiple MMPs that extend beyond MMP-13, including MMP-1, MMP-2, MMP7, MMP-8, MMP-9, and MT1-MMP (43-46). The biologic rationale for the expression of multiple MMPs by MM cells remains unclear, but studies performed to date have most frequently stressed their role in supporting the tissue-trafficking activity of the neoplastic clones as they encounter basement membrane and interstitial matrix barriers in vivo $(44,45)$. While no specific functions have been assigned previously to MM-derived MMP13 , recent studies have proposed roles for the metalloproteinase in promoting tumor-associated osteolytic lesions in breast cancer as well as giant-cell tumors $(15,47-50)$. In each of these cases, however, the preferred mode of MMP-13-dependent activity is the proteolysis of downstream targets thought to play direct or indirect roles in bone resorption, including type I collagen, RANKL, osteoprotegerin, galectin 3 , and TGF- $\beta$ (15, 47-51). By contrast, all of the pro-osteoclastogenic effects associated with the WT proteinase in our studies could be recapitulated by a catalytically inactive E223A MMP-13 mutant. At first glance, the proposition that a catalytically inactive MMP mediates unique biological activities seems counterintuitive. More recently, however, a small subset of MMP family members, including catalytically inactive forms of MMP-3, MMP-9, and MT1-MMP, have been shown to affect a range of cell responses, ranging from cell motility to energy metabolism and cytokine expression (26,
52-55). Interestingly, MT1-MMP has been recently reported to support normal OCL function via a Rac1-dependent process that proceeds independently of the proteolytic activity of the enzyme (55). These latter observations, taken together with those presented here for MMP-13, suggest that OCL function may be modulated by a subset of catalytically inactive MMPs expressed in both a cell-autonomous and non-cell-autonomous fashion. Indeed, consistent with the pro-osteoclastogenic effects of exogenous MMP-13 found in vitro, $M m p 13^{-/-}$OCLs also showed defects in form and function in vitro. Underlining these effects, $\mathrm{Mmp13}^{-/-}$mice have previously been reported to show an osteopetrotic phenotype with increased trabecular bone volume and bone mineral density $(16,56,57)$, along with increased callus bone volume and bone mineral density during active bone remodeling following fracture (10). Given these findings, we considered the possibility that MMP-13 might also affect osteoblast (OB) formation or MM cell behavior itself, as both cell types express the metalloproteinase (Supplemental Figure 17A). However, MMP-13 supplementation did not affect OB differentiation, $\mathrm{MM}$ proliferation, or $\mathrm{MM}$ responses to the antineoplastics bortezomib or pomalidomide (Supplemental Figure 17B and Supplemental Figure 18, respectively). Similarly, MMP-13 silencing in MM cells showed no inhibitory effects on in vitro MM growth or MM-induced OB inhibition in vivo (Supplemental Figure 13C and Supplemental Figure 17C, respectively). 
In light of the finding that MMP-13 induces OCL activation independently of its enzymatic activity, we assessed the effects of MMP-13 inhibition on MM bone lytic lesion and tumor progression by silencing MMP-13 expression, as opposed to using inhibitors directed against its enzymatic activity. Confirming our in vitro studies, MMP-13-KD significantly inhibited the development of bone lytic lesions in vivo. Although MMP-13-KD did not affect MM cell growth during in vitro culture, we observed a modest inhibition of MM tumor growth in the in vivo setting. This is in accordance with data showing that activated OCL cells contribute to adjacent MM growth (20). As such, the inhibition of MM growth in vivo most likely occurs as a consequence of effects secondary to decreased OCL activation within the bone microenvironment following MMP-13 silencing.

In considering the potential mechanisms by which MMP13 regulates osteoclastogenesis in normal as well as neoplastic states, our attention centered on DC-STAMP, a critical fusogenic factor whose deletion undermines OCL formation and function $(32,58)$. Indeed, MMP-13 directly activated both NFATc1 and DC-STAMP expression in developing OCLs. Furthermore, the ability of MMP-13 to promote OCL fusogenesis dovetailed the defects we observed in the maturation of $\mathrm{Mmp13}^{-/-}$myeloid progenitors into multinucleated OCLs that likewise correlated with lower levels of NFATc1 and DC-STAMP expression. With regard to uncovering the MMP-13-dependent signaling cascades that underline OCL progenitor maturation, MMP-13 has been reported to activate ERK1/2 in chondrocytes as well as endothelial cells (35, 59). In our studies, catalytically inactive MMP-13 similarly triggers an ERK1/2-dependent signal transduction cascade that in turn controls NFATc1 and DC-STAMP expression. As surface-bound MMP-13 can be recognized and internalized by the low-density lipoprotein receptor-related protein 1 (i.e., LRP1) (60), we considered the possibility that MMP-13-LRP1-binding interactions induced ERK1/2 phosphorylation. However, both MMP-13dependent ERK1/2 signaling and OCL formation were unaffected when LRP1 function was blocked with the receptor-associated protein (RAP) (data not shown) (35). Efforts are currently underway to identify the MMP-13 receptor(s) that are operative in OCL progenitors, with preliminary cross-linking and mass spectroscopic studies having identified a number of potential cell-surface ligands whose expression will require silencing on a one-by-one basis (our unpublished observation). These studies will, by necessity, need to proceed cautiously, as several MMPs including MMP13 can regulate diverse cell functions by trafficking to the nuclear compartment to exert transcriptional effects $(61,62)$.

To date, all efforts to link MMPs to cancer-induced osteolytic states including MM have emphasized the importance of proteolytic activity and the identification of targeted substrates. Not surprisingly, these studies have also accelerated efforts to identify new therapeutics that can specifically inhibit a single, selected MMP target (63). Our findings, however, support an alternate, and previously unsuspected, role for the MMP-13 zymogen as a MM-derived pro-osteoclastogenic secretagogue. Consonant with our in vitro and mouse model studies, MMP-13 could be detected in the BM compartment of almost all patients with MM in the small cohort of 10 patients studied. While serum MMP-13 levels would not necessarily be predicted to reflect events operative at the OCL-bone interface, we were surprised to find that nearly $60 \%$ of the 18 patients with MM identified with active bone disease who were monitored in our study registered detectable blood levels of MMP-13. At this juncture, we cannot rule out the possibility that the cohort of patients with bony lesions and undetectable serum MMP-13 levels are no longer actively remodeling bone. As such, further studies are underway to monitor serum MMP-13 levels in tandem with markers of active bone turnover. In any case, our findings suggest that, in contrast to interventions designed to target pro-MMP-13-OCL interactions, MMP-13 catalytic activity inhibitors would be predicted to exert little, if any, effect on MM-associated bone disease. As such, efforts designed to identify the MMP-13 receptor as well as the critical MMP-13 domains responsible for receptor binding and signaling may well lead to the development of a new class of bone-sparing therapeutics for the treatment of MM and related bone lytic disease states.

\section{Methods}

Animals. C57BL/6 WT mice were purchased from The Jackson Laboratory. C57BL/6 $\mathrm{Mmp}^{-/-}$mice were maintained as previously described (57). For the 5TGM1 intratibial MM bone disease model, 8- to 10-weekold C57BL/6 Rag2 $2^{-/-}$female mice were purchased from Taconic.

Patients' samples and analysis. BM aspirates were obtained from patients with MM, while serum samples were obtained from either healthy controls or MM patients with or without clinically confirmed (by x-ray or CT) bone disease. MMP-13 levels were determined using a human MMP-13 ELISA kit (EMD Millipore).

For BM and tissue samples, tissue microarray slides (US Biomax) were prepared from formalin-fixed, paraffin-embedded normal and MM tissues. One slide included 21 well-characterized, randomly selected samples from $10 \mathrm{MM}$ patients and 11 normal costal tissues. Five-micrometer sections of 1.5 -mm-diameter cores were stained using a human MMP-13 (hMMP-13) Ab (clone VIIIA2; Calbiochem), combined with Dako EnVision Systems HRP (DAB).

Cells. The human MM cell lines RPMI-8226, H929, and U266 were purchased from the ATCC. MM.1R and OPM2 were provided by Klaus Podar (Dana-Farber Cancer Institute, Boston, Massachusetts, USA). All cell lines were cultured as previously described $(64,65)$. The murine MM cell line 5TGM1-GFP was cultured in IMDM medium with L-glutamine, penicillin-streptomycin, and 10\% FBS. The mouse monocyte/macrophage cell line RAW264.7 and the pre-OB cell line MC3T3-E1 subclone 4 were purchased from the ATCC and cultured in $\alpha$-MEM or $\alpha$-MEM without ascorbic acid (Life Technologies) supplemented with L-glutamine, penicillin-streptomycin, and 10\% FBS, respectively. Mouse primary BMSCs were collected by culturing adherent mouse BM cells in Iscove's modified Dulbecco's medium (IMDM) medium with L-glutamine, penicillin-streptomycin, and 10\% FBS until confluence was reached. Human peripheral blood mononuclear cells (PBMCs) were separated by Ficoll gradient separation, then enriched by Monocyte Enrichment Cocktail (STEMCELL Technologies) according to the manufacturer's instructions (34).

Luminex screening assay. Cell culture supernatants were screened for secreted MMPs (MMP-1,-2,-3,-7,-8, -12, and -13) or cytokines using Luminex assays (Luminex Core Facility at the University of Pittsburgh). The bead set conjugated with a capture $\mathrm{Ab}$ specific to a unique target protein was mixed with a $50-\mu l$ cell culture supernatant sample. 
After washing, secondary biotin-conjugated Ab was added to form a capture sandwich immunoassay. Streptavidin-R-phycoerythrin solution was added and incubated for 30 minutes. A Luminex array reader was used to determine MMP and cytokine concentrations on the basis of 7-point standard curves.

Coculture experiments. Primary BMSCs from patients with MM were seeded in 96-well plates at $4 \times 10^{3}$ cells per well in RPMI 1640 supplemented with $10 \%$ FCS. After 24 hours, human MM cells $\left(4 \times 10^{4}\right.$ cells/well) were seeded on the BMSC layer and cocultured for an additional 48 hours. MMP-13 concentrations in recovered supernatants were determined using a human MMP-13 Fluorokine E Kit (R\&D Systems). To confirm the cell source of MMP-13 in cocultures, human BMSCs were precultured at a concentration of $3 \times 10^{4}$ cells per well in 24 -well plates for 24 hours. GFP ${ }^{+}$RPMI 8226 cells $\left(1 \times 10^{5}\right.$ cells/well $)$ were then seeded onto the stromal cell monolayer for 48 hours. MMP13 expression in RPMI 8226 cells and BMSCs was measured by qRTPCR following the separation of $\mathrm{GFP}^{-}$BMSCs from $\mathrm{GFP}^{+}$RPMI 8266 cells by flow cytometry. $\beta$-actin was used as a loading control.

Transwell assays. BMSCs $\left(3 \times 10^{4}\right.$ cells/well $)$ were cultured for 24 hours in the lower chambers of 24-well Transwell plates. MM cells $\left(1 \times 10^{5}\right.$ cells/well $)$ were seeded in the upper chamber and cocultured for an additional 48 hours. MMP13 mRNA expression in MM cells was measured by qRT-PCR. For MM-OCL Transwell coculture assays, mouse nonadherent BM cells $\left(1.5 \times 10^{6}\right.$ cells/well $)$ were cultured in the lower chambers of 24-well Transwell plates with murine macrophage-CSF (mM-CSF) $(10 \mathrm{ng} / \mathrm{ml})$ and mRANKL (50 ng/ml) (both from R\&D Systems). 5TGM1 EV or 5TGM1-MMP-13-KD cells $\left(3 \times 10^{5}\right.$ cells/ well) were seeded in the upper chamber and cocultured for 4 days.

BMSC CM. BMSCs $\left(5 \times 10^{5}\right)$ isolated from patients with MM were cultured in 100-mm dishes for 24 hours in 10\% FCS-DMEM media. The cells were then washed with PBS and cultured with fresh $5 \%$ FCS-DMEM media for a 3-day incubation period. The CM was collected by centrifugation ( $500 \mathrm{~g}$ for 15 minutes) and cell-free aliquots stored at $-80^{\circ} \mathrm{C}$ until use.

OCL formation and bone resorption assays. For mouse cell studies, $1.5 \times 10^{5}$ nonadherent BM mononuclear cells from C57BL/6 mice (4-6 weeks of age) were cultured in 96-well plates in $\alpha$-MEM supplemented with 10\% FBS, $10 \mathrm{ng} / \mathrm{ml} \mathrm{mM-CSF}$, and $50 \mathrm{ng} / \mathrm{ml}$ mRANKL (R\&D Systems) in the presence or absence of recombinant human (rh) proMMP-1, -2, -9 or -13 (R\&D Systems) for 4 days. Half-media change was carried out on day 3 . The cells were then fixed in formalin and stained for TRAP using a TRAP staining kit (Sigma-Aldrich). TRAP ${ }^{+}$cells containing 3 or more nuclei were counted as OCLs. For bone resorption pit assays, $2 \times 10^{5} \mathrm{BM}$ mononuclear cells were seeded on dentin slices in 96-well plates and treated as described above for 9 days. OCL formation on dentin slices was confirmed by TRAP staining and bone resorption lacunae identified following hematoxylin staining (66). To investigate MMP-13-induced ERK1/2 signaling in pre-OCLs, CD11b+ cells were purified by CD11b microbeads (Miltenyi Biotec) and cultured for 3 days with $10 \mathrm{ng} / \mathrm{ml} \mathrm{M-CSF}$ and $50 \mathrm{ng} / \mathrm{ml} \mathrm{RANKL.} \mathrm{After}$ 4 hours of serum-free starvation, MMP-13 WT or the E223A mutant $(200 \mathrm{ng} / \mathrm{ml})$ was added for the durations indicated in Supplemental Figure 12A prior to collection of lysates for Western blot analysis.

For human cell studies, BM mononuclear cells $\left(1 \times 10^{5}\right.$ cells/ well) isolated from patients with MM were seeded in 96-well plates in $\alpha$-MEM supplemented with $20 \%$ horse serum, $10 \mathrm{ng} / \mathrm{ml}$ human $\mathrm{M}-\mathrm{CSF}$ (hM-CSF), and $50 \mathrm{ng} / \mathrm{ml}$ soluble human RANKL (hRANKL) (both from R\&D Systems) in the absence or presence of rh-pro-MMP13. Half-media changes were carried out every 3 days and OCL formation assessed by staining with mAb 23c6 (CD51/61), which was provided by Michael Horton (Rayne Institute, Bone and Mineral Center, London, United Kingdom) using a VECTASTAIN ABC-AP Kit (Vector Laboratories) (66). For bone resorption pit assays, $2 \times 10^{5} \mathrm{BM}$ mononuclear cells were seeded on dentin slices in 96-well plates and treated as described above for 21 days. The number of OCLs per well, the number of nuclei per OCL, and OCL size were quantified using an Olympus IX70 microscope equipped with a 20/0.40 numeric aperture objective lens and SPOT software (Diagnostic Instruments) or a Leica DMI 6000B fluorescence microscope equipped with an HCX PL S-APO 10×/NA 0.3 objective lens, coupled with a Leica DFC365 FX digital camera and operated by LAS AF 6.2 software (Leica Microsystems). Images of resorption lacunae were captured using an OMAX 40X-2000X Digital Microscope with ScopeImage 9.0 software (BioImager) or Magnafire 4.1 software (Optronics).

OB differentiation. MC3T3 E1 cells $\left(1 \times 10^{4}\right)$ were seeded in 24-well culture dishes in OB differentiation media ( $\alpha$-MEM without ascorbic acid, supplemented with $10 \%$ FBS, $1 \%$ penicillin-streptomycin, $50 \mu \mathrm{g} /$ $\mathrm{ml} \mathrm{L}$-ascorbic acid, and $2 \mathrm{mM} \beta$-glycerophosphate), with or without MMP-13 (200 ng/ml), for up to 14 days. Media were changed every 3 days, and, at the times indicated in Supplemental Figure 17B, cultures were fixed in $4 \%$ paraformaldehyde for 10 minutes and stained for alkaline phosphatase or alizarin red.

Immunoblot analysis. Immunoblotting was performed as described previously $(64,67)$ using the following Abs: anti-MMP-13 (ab39012; Abcam); anti-DC-STAMP (clone 1A2; EMD Millipore); anti-NFATc1 (7A6) and anti-TRAF6 (H274) (both from Santa Cruz Biotechnology Inc.); anti-ubiquination (Ubi-1; Thermo Fisher Scientific); anti-phosphorylated-c-Raf (anti-p-c-Raf) (Ser338) (56A6), anti-p-MEK1/2 (Ser217/221), anti-p-ERK1/2 (D13.14.4E), anti-p-p90RSK (Ser380) (D3H11), and anti-ERK2 (9108) (all from Cell Signaling Technology); and $\beta$-actin (Sigma-Aldrich).

qRT-PCR analysis. Total RNA was isolated with TRIzol reagent (Thermo Fisher Scientific) and cDNA generated with SuperScript III reverse transcriptase (Invitrogen), followed by SYBR Green-based (Invitrogen) qRT-PCR assays performed as previously described (64). Primer pairs were as follows: human MMP13: forward, 5'-GATGGGCCCTCTGGCCTGCT; reverse, 5'-TGGCCGAACTCATGCGCAGC; human $\beta$-actin: forward, 5'-CGAGCACAGAGCCTCGCCTTTG; reverse, 5'-CGACGAGCGCGGCGATATCAT; mouse Mmp13: forward, 5'-GGTCCAGGCGATGAAGACCCC; reverse, 5'-GGGTGCAGGCGCCAGAAGAA; and mouse $\beta$-actin: forward, 5'-AACCCTAAGGCCAACCGTGAAA; reverse, 5'-CGACCAGAGGCATACAGGGACAG. Mouse Nfatc1 and Dcstamp mRNA were analyzed by TaqMan qRT-PCR (Life Technologies).

Flow cytometric assay. For flow cytometric analysis, human PBMCs or mouse OCLs were harvested, washed once with FACS buffer (HBSS buffer supplemented with 0.5\% BSA and 0.1\% NaN3), and incubated with primary Ab mouse anti-DC-STAMP clone 1A2 (EMD Millipore) and Fc receptor blocker anti-CD16/CD32 (BD Pharmingen) at room temperature for 20 minutes. Cells were rewashed with FACS buffer and finally incubated with FITC-conjugated goat antimouse secondary Ab (Invitrogen) at room temperature for 20 minutes. FACS data were acquired using a BD FACSCanto System and analyzed by FlowJo software $(34,67)$. 
Expression constructs and site-directed mutagenesis. WT human MMP13 cDNA was generated by PCR using CDNA prepared from RPMI 8266 cells as a template with the following primers: forward, 5'-GACTGGAATTCACCATGCATCCAGGGGTCCTGGCT-3'; reverse, 5'-GACTCTCGAGGCACACCACAAAATGGAATTTGC-3'. PCR products were then cloned into pcDNA 6/myc-His B (Invitrogen) after EcoRI-XhoI (New England BioLabs) double digestion. For generation of the MMP-13 E223A mutant, site-directed mutagenesis PCR was performed with the pcDNA6/myc-His B-MMP-13 WT construct as a template using the following primers: forward, 5'-CTTGTTGCTGCGCATGCATTCGGCCACTCCTTA-3'; reverse, 5'-TAAGGAGTGGCCGAATGCATGCGCAGCAACAAG-3'. Similarly, MMP-13 procatalytic domain (aa 1-267) constructs were generated by PCR using pcDNA6/myc-His B-MMP-13 WT as a template with the following primers: forward, 5'-GACTGGAATTCACCATGCATCCAGGGGTCCTGGCT-3'; reverse, 5'-GACTCTCGAGACCATAGAGAGACTGGAT-3', followed by cloning into pcDNA 6/myc-His A after EcoRI-XhoI double digestion. An MMP-13 hemopexin domain (deletion 37-267) construct was generated by mutagenesis PCR with a pcDNA6/myc-His B-MMP-13 WT construct as a template and the following primers: forward, 5'-TCTGAGGAAGACCTCCCGGGAGATGAAGACCCCAAC-3'; reverse, 5'-GTTGGGGTCTTCATCTCCCGGGAGGTCTTCCTCAGA-3'. Constructs were verified by sequencing and MMP-13 mutant protein expression confirmed by Western blotting following transfection in HEK-293 cells.

Lentivirus infection and MMP-13 knockdown and knockin. To silence MMP-13 expression in mouse MM cells, 5TGM1-GFP cells were transduced with an EV lentiviral control or an MMP-13-targeting shRNA lentiviral construct 1 (5'-CCGGGCCTTACATTTGGCTTAATTTCTCGAGAAATTAAGCCAAATGTAAGGCTTTTTG-3') or an MMP-13-targeting shRNA lentiviral construct 2 (5'-CCGGGCACTACTTGAAATCATACTACTCGAGTAGTATGATTTCAAGTAGTGCTTTTTG-3') (provided by Robert W. Sobol, Hillman Cancer Center, Pittsburgh, Pennsylvania, USA). 5TGM1-GFP cells were incubated with lentiviral particles and polybrene $(8 \mu \mathrm{g} / \mathrm{ml}$ for 16 hours $)$ and then washed. A second infection was repeated 24 hours later. Cells were selected by culturing for 14 days in puromycin $(8 \mu \mathrm{g} / \mathrm{ml})$. MMP-13 protein and mRNA levels were determined in control and silenced cells by Western blotting and qRT-PCR, respectively. To knock MMP-13 WT or the E223A mutant into 5TGM1-GFP MMP-13-KD cells, MMP-13 WT or the E223A mutant was inserted into a pLHCX retroviral vector (Clontech). Retroviruses were packed by Phoenix-AMPHO cells (ATCC), and 5TGM1GFP MMP-13-KD cells were transduced as described above. Cells were selected by culturing for 14 days in hygromycin B (100 $\mu \mathrm{g} / \mathrm{ml})$. MMP-13 protein was determined by Western blot analysis.

Protein purification. pcDNA6/myc-His B-MMP-13 WT, MMP$13 \mathrm{E} 223 \mathrm{~A}$, as well as MMP-13 procatalytic domain (aa 1-267) and hemopexin domain (deletion aa 37-267) constructs were transfected into HEK-293 cells using Lipofectamine 2000 (Invitrogen) and cultured in Opti-MEM serum-free medium (Thermo Fisher Scientific). Cell culture media were collected 48 hours after transfection, concentrated by ultrafiltration, and incubated with Ni-NTA agarose (QIAGEN) at $4^{\circ} \mathrm{C}$ for 2 hours. The tagged proteins were washed, eluted according to the manufacturer's protocol, de-salted by ultrafiltration, and resuspended in PBS. Protein purity and concentration were determined by Coomassie Brilliant Blue R-250 staining following SDS-PAGE and a Bradford protein assay, respec- tively. The proteolytic activity of WT and E223A mutant MMP-13 was determined with an MMP-13 Fluorokine E Kit (R\&D Systems) after pretreatment with $1 \mathrm{mM} \mathrm{4-aminophenylmercuric} \mathrm{acetate)}$ (Sigma-Aldrich) at $37^{\circ} \mathrm{C}$ for 1 hour, according to the manufacturer's instructions, and its activity compared with commercially obtained MMP-13 protein (R\&D Systems) as an internal standard. In all cell cultures, MMP-13 was used at a concentration of $200 \mathrm{ng} / \mathrm{ml}$ unless stated otherwise.

${ }^{3} \mathrm{H}$-thymidine incorporation assay. 5TGM1-GFP-EV or MMP-13KD cells $\left(3 \times 10^{4}\right.$ cells $)$ were incubated in 96-well plates in $200 \mu \mathrm{l}$ IMDM containing $10 \% \mathrm{FBS}$ at $37^{\circ} \mathrm{C}$ and $5 \% \mathrm{CO}_{2}$ for 2 days. DNA synthesis was measured by ${ }^{3} \mathrm{H}$-thymidine incorporation (NEN Products, PerkinElmer Life and Analytical Sciences). Cells were pulsed with ${ }^{3} \mathrm{H}$-thymidine $(1 \mu \mathrm{Ci} /$ well or $0.037 \mathrm{MBq})$ for the last 8 hours of culture, harvested onto glass fiber filter mats (Wallac) using an automatic cell harvester (Harvester 96, Mach III; Tomtec), and counted using a Wallac TriLux Beta Plate Scintillation Counter (PerkinElmer Life and Analytical Sciences) (64).

Cell proliferation assay. MM cells $\left(1 \times 10^{4}\right)$ or $\mathrm{CD}_{11 \mathrm{~b}^{+}}$mouse $\mathrm{BM}$ mononuclear cells $\left(1 \times 10^{5}\right)$ were incubated in 96-well plates in $100 \mu \mathrm{l}$ culture medium with bortezomib $(100 \mathrm{nM})$, pomalidomide $(2 \mu \mathrm{M})$, or vehicle, with or without MMP-13 $(200 \mathrm{ng} / \mathrm{ml})$, at $37^{\circ} \mathrm{C}$ and $5 \% \mathrm{CO}_{2}$ for up to 3 days. DMSO was added in the control group. Ten microliters of Premixed WST-1 Cell Proliferation Reagent (Clontech, Takara Bio) was added to each well for 4 hours and absorption determined in an ELISA reader (BioTek Instruments).

Collagen zymography. MM cells, RAW264.7 cells, MC3T3-E1 clone 4 , or mouse primary BMSCs were cultured in standard medium until confluent and then switched to Opti-MEM I serum-free medium (Thermo Fisher Scientific) for 3 days. Medium was collected and concentrated by Amicon ultracentrifugal filtration (EMD Millipore). The concentrated supernatants were analyzed by $10 \%$ SDS-PAGE gel containing $0.5 \%$ collagen type I as previously described, and gels were stained with Coomassie Brilliant Blue R250 (Thermo Fisher Scientific) and then de-stained (68). In selected experiments, $0.4 \mu \mathrm{g}$ purified MMP-13 WT or E223A mutant protein was incubated in MMP-13 reaction buffer, with or without $1 \mathrm{mM}$ APMA, for 20 minutes at $37^{\circ} \mathrm{C}$ and analyzed by collagen zymography. The molecular weight of proMMP-13 is $60 \mathrm{kD}$, while that of activated MMP-13 is $48 \mathrm{kD}$ (69).

Mouse MM model. 5TGM1-GFP EV or MMP-13-KD cells $\left(1 \times 10^{5}\right.$ cells) were bilaterally injected into both tibiae of C57BL/6 Rag2 ${ }^{-1-}$ female mice as described previously $(41,70)$. Mice were sacrificed 4 weeks later, and tibiae were collected for GFP imaging and then fixed in $4 \%$ neutral-buffered formalin for micro-qCT analysis. Following micro-qCT scanning, the same tibiae were decalcified with $10 \%$ EDTA and subjected to histologic analysis. Tumor burden was assessed by serum analysis of the myeloma-specific IgG2b.

Micro-qCT analysis. Mice tibiae were dissected 4 weeks after tumor injection and fixed in 10\% neutral-buffered formalin buffer for 2 days. Trabecular bone from the proximal tibia and cortical bone at the tibia midshaft were scanned using a micro-qCT system (VivaCT 40; Scanco Medical). For trabecular microstructure, 100 slices, corresponding to a $1.05-\mathrm{mm}$ region underneath the growth plate, were obtained with $10.5 \mu \mathrm{m}$ spatial resolution. For the cortical bone, 50 slices, corresponding to a $1.05-\mathrm{mm}$ region starting in a region that was $56 \%$ of the tibia length from its proximal end, were obtained with 21 $\mu \mathrm{m}$ spatial resolution. A global threshold technique was applied to seg- 
ment the grayscale image into binarized images. Both the trabecular and cortical compartments were analyzed with a semiautomatic contouring technique to assess microstructural parameters (71).

Bone histology and immunohistochemical staining. Following micro-qCT, the same tibiae were decalcified in 10\% EDTA (pH 7.4) buffer for 2 weeks at $4{ }^{\circ} \mathrm{C}$ and embedded in paraffin. Bone sections (5 $\mu \mathrm{m}$ thickness) were stained with $\mathrm{H} \& \mathrm{E}$ and for tartrate-resistant acid phosphatase (TRAP) using a Leukocyte Acid Phosphatase Kit (SigmaAldrich). For OB staining, paraffin sections underwent $10 \mathrm{mM}$ sodium citrate antigen retrieval, followed by staining with anti-osteocalcin $\mathrm{Ab}$ (ab93876; Abcam) and EnVision ${ }^{+}$Systems HRP (DAB) (Dako) according to the manufacturer's instructions.

Statistics. Quantitative data are presented as the mean \pm SD or SEM, as indicated in the figure legends. Statistical significance was assessed by a 2-tailed Student's $t$ test or a Wilcoxon rank-sum test, as appropriate, for comparisons between 2 groups. In experiments with more than 2 experimental groups, Westfall's adjustment for multiple comparisons (72) was applied to pairwise comparisons following ANOVA. Count data were analyzed by Poisson ANOVA (73), with a similar adjustment for multiple comparisons. The relationship between quantitative variables was characterized by linear or Poisson regression, depending on the nature of the response variable. A $P$ value of 0.05 or less was considered significant, and a $P$ of 0.01 or less was considered highly significant.

Study approval. All animal procedures were reviewed and approved by the IACUC of Columbia University (protocols AC-AAAE9803 and AC-AAAN5201). BM aspirates were obtained with informed consent from healthy controls and MM patients under an IRB-approved tissue repository protocol at the University of Pittsburgh Cancer Institute (UPCI 86-22) and Columbia University Medical Center (AAAL9054).

\section{Author contributions}

JF, SL, and RF conducted experiments and analyzed data. HM and FS contributed mouse BM samples. JW, SR, and XEG conducted micro-qCT scanning and morphologic analysis of mice tibiae. TL helped with IHC staining of slides of the patients' samples. DN performed statistical analysis of the data. GDR and MYM contributed vital reagents and critically revised the manuscript. JF, SJW, and SL designed the research studies, analyzed data, and wrote the manuscript.

\section{Acknowledgments}

This work was supported by a 2013 American Society of Hematology (ASH) Bridge Grant Award (to S. Lentzsch); NIH grants (R01 CA175313, to S. Lentzsch; R01 HL93716, to M.Y. Mapara; and R21 CA141426, R01 AR059679, and R21 CA179017-01, to G.D. Roodman); VA Merit Review funds (to G.D. Roodman), and a National Cancer Institute (NCI) award (P30CA047904, to D. Normolle).

Address correspondence to: Suzanne Lentzsch, Department of Medicine, Division of Hematology/Oncology, College of Physicians and Surgeons, Columbia University, Herbert Irving Pavilion, R 953, 161 Ft. Washington Ave., New York, New York 10032, USA. Phone: 646.317.4840; E-mail: s13440@columbia.edu.
1. Roodman GD. Pathogenesis of myeloma bone disease. J Cell Biochem. 2010;109(2):283-291.

2. Lentzsch S, Ehrlich LA, Roodman GD. Pathophysiology of multiple myeloma bone disease. Hematol Oncol Clin North Am. 2007;21(6):1035-1049.

3. Raje N, Roodman GD. Advances in the biology and treatment of bone disease in multiple myeloma. Clin Cancer Res. 2011;17(6):1278-1286.

4. Farrugia AN, et al. Receptor activator of nuclear factor-kappaB ligand expression by human myeloma cells mediates osteoclast formation in vitro and correlates with bone destruction in vivo. Cancer Res. 2003;63(17):5438-5445.

5. Han JH, Choi SJ, Kurihara N, Koide M, Oba Y, Roodman GD. Macrophage inflammatory protein-1alpha is an osteoclastogenic factor in myeloma that is independent of receptor activator of nuclear factor kappaB ligand. Blood. 2001;97(11):3349-3353.

6. Lee JW, et al. IL-3 expression by myeloma cells increases both osteoclast formation and growth of myeloma cells. Blood. 2004;103(6):2308-2315.

7. Roodman GD. Treatment strategies for bone disease. Bone Marrow Transplant. 2007;40(12):1139-1146.

8. Page-McCaw A, Ewald AJ, Werb Z. Matrix metalloproteinases and the regulation of tissue remodelling. Nat Rev Mol Cell Biol. 2007;8(3):221-233.

9. Rowe RG, Weiss SJ. Navigating ECM barriers at the invasive front: the cancer cell-stroma interface. Annu Rev Cell Dev Biol. 2009;25:567-595.

10. Behonick DJ, et al. Role of matrix metalloproteinase 13 in both endochondral and intramembranous ossification during skeletal regeneration.
PLoS One. 2007;2(11):e1150.

11. Borzi RM, et al. Matrix metalloproteinase 13 loss associated with impaired extracellular matrix remodeling disrupts chondrocyte differentiation by concerted effects on multiple regulatory factors. Arthritis Rheum. 2010;62(8):2370-2381.

12. Juncker-Jensen A, Lund LR. Phenotypic overlap between MMP-13 and the plasminogen activation system during wound healing in mice. PLoS One. 2011;6(2):e16954.

13. Lederle W, et al. MMP13 as a stromal mediator in controlling persistent angiogenesis in skin carcinoma. Carcinogenesis. 2010;31(7):1175-1184.

14. Nielsen BS, et al. Collagenase-3 expression in breast myofibroblasts as a molecular marker of transition of ductal carcinoma in situ lesions to invasive ductal carcinomas. Cancer Res. 2001;61(19):7091-7100.

15. Shah M, et al. An MMP13-selective inhibitor delays primary tumor growth and the onset of tumor-associated osteolytic lesions in experimental models of breast cancer. PLoS One. 2012;7(1):e29615.

16. Tang SY, Herber RP, Ho SP, Alliston T. Matrix metalloproteinase- 13 is required for osteocytic perilacunar remodeling and maintains bone fracture resistance. J Bone Miner Res. 2012;27(9):1936-1950.

17. Toriseva M, et al. MMP-13 regulates growth of wound granulation tissue and modulates gene expression signatures involved in inflammation, proteolysis, and cell viability. PLoS One. 2012;7(8):e42596.

18. Zigrino $\mathrm{P}$, et al. Stromal expression of MMP-13 is required for melanoma invasion and metastasis. J Invest Dermatol. 2009;129(11):2686-2693.

19. Zijlstra A, et al. Collagenolysis-dependent angiogenesis mediated by matrix metalloproteinase-13 (collagenase-3). J Biol Chem. 2004;279(26):27633-27645.

20. Hideshima T, Mitsiades C, Tonon G, Richardson PG, Anderson KC. Understanding multiple myeloma pathogenesis in the bone marrow to identify new therapeutic targets. Nat Rev Cancer. 2007;7(8):585-598.

21. Markovina S, et al. Bone marrow stromal cells from multiple myeloma patients uniquely induce bortezomib resistant NF- $\mathrm{KB}$ activity in myeloma cells. Mol Cancer. 2010;9(1):176.

22. Nyman JS, et al. Differential effects between the loss of MMP-2 and MMP-9 on structural and tissue-level properties of bone. JBone Miner Res. 2011;26(6):1252-1260.

23. Reiter LA, et al. Pyran-containing sulfonamide hydroxamic acids: potent MMP inhibitors that spare MMP-1. Bioorg Med Chem Lett. 2004;14(13):3389-3395.

24. Engel CK, et al. Structural basis for the highly selective inhibition of MMP-13. Chem Biol. 2005;12(2):181-189.

25. Kennedy AM, et al. MMP13 mutation causes spondyloepimetaphyseal dysplasia, Missouri type (SEMD(MO)). JClin Invest. 2005;115(10):2832-2842.

26. Correia AL, Mori H, Chen EI, Schmitt FC, Bissell MJ. The hemopexin domain of MMP3 is responsible for mammary epithelial invasion and morphogenesis through extracellular interaction with 
HSP9Obeta. Genes Dev. 2013;27(7):805-817.

27. Dufour A, et al. Small-molecule anticancer compounds selectively target the hemopexin domain of matrix metalloproteinase-9. Cancer Res. 2011;71(14):4977-4988.

28. Ezhilarasan R, Jadhav U, Mohanam I, Rao JS, Gujrati M, Mohanam S. The hemopexin domain of MMP-9 inhibits angiogenesis and retards the growth of intracranial glioblastoma xenograft in nude mice. Int J Cancer. 2009;124(2):306-315.

29. Mantuano $E$, et al. The hemopexin domain of matrix metalloproteinase-9 activates cell signaling and promotes migration of schwann cells by binding to low-density lipoprotein receptor-related protein. J Neurosci. 2008;28(45):11571-11582.

30. Van den Steen PE, et al. The hemopexin and O-glycosylated domains tune gelatinase B/MMP-9 bioavailability via inhibition and binding to cargo receptors. J Biol Chem. 2006;281(27):18626-18637.

31. Kukita T, et al. RANKL-induced DC-STAMP is essential for osteoclastogenesis. JExp Med. 2004;200(7):941-946.

32. Yagi M, et al. Induction of DC-STAMP by alternative activation and downstream signaling mechanisms. J Bone Miner Res. 2007;22(7):992-1001.

33. Mensah KA, Ritchlin CT, Schwarz EM. RANKL induces heterogeneous DC-STAMP(lo) and DC-STAMP(hi) osteoclast precursors of which the DC-STAMP(lo) precursors are the master fusogens. J Cell Physiol. 2010;223(1):76-83.

34. Chiu YG, Ritchlin CT. Characterization of DC-STAMP+ Cells in Human Bone Marrow. J Bone Marrow Res. 2013;1:1000127.

35. Raggatt LJ, Jefcoat SC Jr, Choudhury I, Williams S, Tiku M, Partridge NC. Matrix metalloproteinase-13 influences ERK signalling in articular rabbit chondrocytes. Osteoarthritis Cartilage. 2006;14(7):680-689.

36. Lee MS, et al. GM-CSF regulates fusion of mononuclear osteoclasts into bone-resorbing osteoclasts by activating the Ras/ERK pathway. JImmunol. 2009;183(5):3390-3399.

37. Lamothe B, Webster WK, Gopinathan A, Besse A, Campos AD, Darnay BG. TRAF6 ubiquitin ligase is essential for RANKL signaling and osteoclast differentiation. Biochem Biophys Res Commun. 2007;359(4):1044-1049.

38. Bradley EW, Ruan MM, Vrable A, Oursler MJ. Pathway crosstalk between Ras/Raf and PI3K in promotion of M-CSF-induced MEK/ERKmediated osteoclast survival. JCell Biochem. 2008;104(4):1439-1451.

39. He Y, et al. Erk1 positively regulates osteoclast differentiation and bone resorptive activity. PLoS One. 2011;6(9): 24780.

40. Favata MF, et al. Identification of a novel inhibitor of mitogen-activated protein kinase kinase. J Biol Chem. 1998;273(29):18623-18632.

41. Fowler JA, Mundy GR, Lwin ST, Lynch CC, Edwards CM. A murine model of myeloma that allows genetic manipulation of the host microenvironment. Dis Model Mech. 2009;2(11-12):604-611.

42. Roodman GD. Mechanisms of bone metastasis.
N EnglJ Med. 2004;350(16):1655-1664.

43. Wahlgren J, et al. Expression and induction of collagenases (MMP-8 and -13) in plasma cells associated with bone-destructive lesions. J Pathol. 2001;194(2):217-224.

44. Van Valckenborgh E, et al. Multifunctional role of matrix metalloproteinases in multiple myeloma: a study in the 5T2MM mouse model. Am J Pathol. 2004;165(3):869-878.

45. Parmo-Cabanas M, et al. Role of metalloproteinases MMP-9 and MT1-MMP in CXCL12-promoted myeloma cell invasion across basement membranes. J Pathol. 2006;208(1):108-118.

46. Alsayed Y, et al. Mechanisms of regulation of CXCR4/SDF-1 (CXCL12)-dependent migration and homing in multiple myeloma. Blood. 2007;109(7):2708-2717.

47. Mak IW, et al. Evidence for the role of matrix metalloproteinase-13 in bone resorption by giant cell tumor of bone. Hum Pathol. 2010;41(9):1320-1329.

48. Mak IW, Turcotte RE, Popovic S, Singh G, Ghert M. AP- 1 as a regulator of MMP-13 in the stromal cell of giant cell tumor of bone. Biochem Res Int. 2011;2011:164197.

49. Nannuru KC, Futakuchi M, Varney ML, Vincent TM, Marcusson EG, Singh RK. Matrix metalloproteinase (MMP)-13 regulates mammary tumor-induced osteolysis by activating MMP9 and transforming growth factor-beta signaling at the tumor-bone interface. Cancer Res. 2010;70(9):3494-3504.

50. Pivetta E, et al. MMP-13 stimulates osteoclast differentiation and activation in tumour breast bone metastases. Breast Cancer Res. 2011;13(5):R105.

51. Morrison C, Mancini S, Cipollone J, Kappelhoff R, Roskelley C, Overall C. Microarray and proteomic analysis of breast cancer cell and osteoblast co-cultures: role of osteoblast matrix metalloproteinase (MMP)-13 in bone metastasis. J Biol Chem. 2011;286(39):34271-34285.

52. Dufour A, Zucker S, Sampson NS, Kuscu C, Cao J. Role of matrix metalloproteinase-9 dimers in cell migration: design of inhibitory peptides. J Biol Chem. 2010;285(46):35944-35956.

53. Gonzalo P, et al. MT1-MMP is required for myeloid cell fusion via regulation of Rac1 signaling. Dev Cell. 2010;18(1):77-89.

54. Sakamoto T, Seiki M. A membrane protease regulates energy production in macrophages by activating hypoxia-inducible factor-1 via a non-proteolytic mechanism. J Biol Chem. 2010;285(39):29951-29964.

55. Shimizu-Hirota R, et al. MT1-MMP regulates the PI3Kdelta.Mi-2/NuRD-dependent control of macrophage immune function. Genes Dev. 2012;26(4):395-413

56. Stickens D, et al. Altered endochondral bone development in matrix metalloproteinase 13-deficient mice. Development. 2004;131(23):5883-5895.

57. Inada $\mathrm{M}$, et al. Critical roles for collagenase-3 (Mmp13) in development of growth plate cartilage and in endochondral ossification. Proc Natl
Acad Sci U S A. 2004;101(49):17192-17197.

58. Yagi M, et al. DC-STAMP is essential for cell-cell fusion in osteoclasts and foreign body giant cells. JExp Med. 2005;202(3):345-351.

59. Kudo Y, et al. Matrix metalloproteinase-13 (MMP-13) directly and indirectly promotes tumor angiogenesis. J Biol Chem. 2012;287(46):38716-38728.

60. Barmina OY, et al. Collagenase- 3 binds to a specific receptor and requires the low density lipoprotein receptor-related protein for internalization. J Biol Chem. 1999;274(42):30087-30093.

61. Marchant DJ, et al. A new transcriptional role for matrix metalloproteinase-12 in antiviral immunity. Nat Med. 2014;20(5):493-502.

62. Cuadrado E, et al. Matrix metalloproteinase-13 is activated and is found in the nucleus of neural cells after cerebral ischemia. JCereb Blood Flow Metab. 2009;29(2):398-410.

63. Dufour A, Overall CM. Missing the target: matrix metalloproteinase antitargets in inflammation and cancer. Trends Pharmacol Sci. 2013;34(4):233-242.

64. Li S, et al. IMiD immunomodulatory compounds block C/EBP $\{$ beta\} translation through eIF $4 \mathrm{E}$ down-regulation resulting in inhibition of MM. Blood. 2011;117(19):5157-5165.

65. Pal R, et al. C/EBP $\beta$ regulates transcription factors critical for proliferation and survival of multiple myeloma cells. Blood. 2009;114(18):3890-3898.

66. Feng R, et al. SDX-308, a nonsteroidal antiinflammatory agent, inhibits NF- $\mathrm{\kappa B}$ activity, resulting in strong inhibition of osteoclast formation/activity and multiple myeloma cell growth. Blood. 2007;109(5):2130-2138.

67. Li S, Fu J, Ma H, Mapara MY, Lentzsch S. Lenalidomide-induced upregulation of CXCR4 in CD $34^{+}$ hematopoietic cells, a potential mechanism of decreased hematopoietic progenitor mobilization. Leukemia. 2013;27(6):1407-1411.

68. Gogly B, Groult N, Hornebeck W, Godeau G, Pellat B. Collagen zymography as a sensitive and specific technique for the determination of subpicogram levels of interstitial collagenase. Anal Biochem. 1998;255(2):211-216.

69. Knauper V, et al. Cellular mechanisms for human procollagenase-3 (MMP-13) activation. Evidence that MT1-MMP (MMP-14) and gelatinase a (MMP-2) are able to generate active enzyme. JBiol Chem. 1996;271(29):17124-17131.

70. D'Souza S, et al. Gfi1 expressed in bone marrow stromal cells is a novel osteoblast suppressor in patients with multiple myeloma bone disease. Blood. 2011;118(26):6871-6880.

71. Kajimura D, et al. Adiponectin regulates bone mass via opposite central and peripheral mechanisms through FoxO1. Cell Metab. 2013;17(6):901-915.

72. Westfall PH. Multiple testing of general contrasts using logical constraints and correlations. JASA. 1997;92(437):299-306.

73. McCullagh P, Nelder JA. Generalized Linear Models. London, United Kingdom: Chapman and Hall; 1989. 